\title{
Stability and Bifurcation Analysis for a Delay Differential Equation of Hepatitis B Virus Infection
}

\author{
Xinchao Yang, ${ }^{1}$ Xiju Zong, ${ }^{2}$ Xingong Cheng, ${ }^{2}$ and Zhenlai Han ${ }^{3}$ \\ ${ }^{1}$ School of Medical and Life Science, University of Jinan, Jinan, Shandong 250022, China \\ ${ }^{2}$ School of Electrical Engineering, University of Jinan, Jinan, Shandong 250022, China \\ ${ }^{3}$ School of Science, University of Jinan, Jinan, Shandong 250022, China
}

Correspondence should be addressed to Xiju Zong; cse_zongxj@ujn.edu.cn

Received 29 July 2012; Accepted 27 December 2012

Academic Editor: Yongkun Li

Copyright (c) 2013 Xinchao Yang et al. This is an open access article distributed under the Creative Commons Attribution License, which permits unrestricted use, distribution, and reproduction in any medium, provided the original work is properly cited.

\begin{abstract}
The stability and bifurcation analysis for a delay differential equation of hepatitis B virus infection is investigated. We show the existence of nonnegative equilibria under some appropriated conditions. The existence of the Hopf bifurcation with delay $\tau$ at the endemic equilibria is established by analyzing the distribution of the characteristic values. The explicit formulae which determine the direction of the bifurcations, stability, and the other properties of the bifurcating periodic solutions are given by using the normal form theory and the center manifold theorem. Numerical simulation verifies the theoretical results.
\end{abstract}

\section{Introduction}

Recently, a hepatitis B virus (HBV) model with time delay that was proposed and investigated in the literature [1-4] caught the attention of a lot of mathematicians. In practice, the HBV model has suffered time delay caused by the HBV incubation period, which varies from 45 to 180 days, and the delay in viral shedding which both suggest that viral production delay may significantly impact infection dynamics [1]. Precisely, the HBV model with time delay reads as the following:

$$
\begin{aligned}
\frac{\mathrm{d} x(t)}{\mathrm{d} t}= & \lambda-d x(t)-\frac{\beta x(t) v(t)}{x(t)+e(t)+y(t)}, \\
\frac{\mathrm{d} e(t)}{\mathrm{d} t}= & -d e(t)+\frac{\beta x(t) v(t)}{x(t)+e(t)+y(t)} \\
& -\frac{\beta e^{-d \tau} x(t-\tau) v(t-\tau)}{x(t-\tau)+e(t-\tau)+y(t-\tau)}, \\
\frac{\mathrm{d} y(t)}{\mathrm{d} t}= & \frac{\beta e^{-d \tau} x(t-\tau) v(t-\tau)}{x(t-\tau)+e(t-\tau)+y(t-\tau)}-a y(t),
\end{aligned}
$$

$$
\frac{\mathrm{d} v(t)}{\mathrm{d} t}=k y(t)-\mu v(t),
$$

where $x(t)$ and $y(t)$ represent the number of uninfected cells and infected cells, respectively. $e(t)$ represents the number of exposed cells, that is, the cells that have acquired the virus but are not yet producing new virions. $v(t)$ denotes the number of free virions. $\tau$ is the time delay for virion production. Here, the positive constant $\lambda$ is the rate at which new uninfected live cells are generated. The positive constant $d$ is the per capita death rate of uninfected live cells. Infected live cells are killed by immune cells at rate $a y$ and produce free virions at rate $k y$, where $k$ is what so-called "burst" constant. Free virions are cleared by lymphatic and other mechanisms at rate $\mu v$, where $\mu$ is a constant. $\beta>0$ is an incidence rate coefficient describing the infection process. The initial conditions for the system (1) are

$$
\begin{gathered}
x(s)=x_{0}(s), \quad y(s)=y_{0}(s), \\
v(s)=v_{0}(s), \quad s \in[-\tau, 0], \\
E_{0}=\beta \int_{-\tau}^{0} \frac{e^{d s} v_{0}(s) x_{0}(s)}{x_{0}(s)+y_{0}(s)+e_{0}(s)} \mathrm{d} s,
\end{gathered}
$$


where $x_{0}, y_{0}$, and $v_{0}$ are nonnegative functions. Based on some observations of virus particles $v$, the system (1) is simplified in [1] as the following:

$$
\begin{gathered}
\frac{\mathrm{d} x(t)}{\mathrm{d} t}=\lambda-d x(t)-\frac{\beta k x(t) y(t)}{\mu[x(t)+y(t)]}, \\
\frac{\mathrm{d} e(t)}{\mathrm{d} t}=-d e(t)+\frac{\beta k x(t) y(t)}{\mu[x(t)+y(t)]} \\
-\frac{\beta k e^{-d \tau} x(t-\tau) y(t-\tau)}{\mu[x(t-\tau)+y(t-\tau)]}, \\
\frac{\mathrm{d} y(t)}{\mathrm{d} t}=\frac{\beta k e^{-d \tau} x(t-\tau) y(t-\tau)}{\mu[x(t-\tau)+y(t-\tau)]}-a y(t) .
\end{gathered}
$$

A direct computation shows that the basic infection reproduction number for the system (2) is

$$
R_{0}=\frac{\beta k e^{-d \tau}}{a \mu} .
$$

For the sake of simplicity, let $\bar{x}=x-(\lambda / d)$, and the system (3) is equivalent to the following system:

$$
\begin{gathered}
\frac{\mathrm{d} \bar{x}(t)}{\mathrm{d} t}=-d \bar{x}(t)-\frac{\beta k[\bar{x}(t)+(\lambda / d)] y(t)}{\mu[\bar{x}(t)+(\lambda / d)+y(t)]} \\
\frac{\mathrm{d} e(t)}{\mathrm{d} t}=-d e(t)+\frac{\beta k[\bar{x}(t)+(\lambda / d)] y(t)}{\mu[\bar{x}(t)+(\lambda / d)+y(t)]} \\
-\frac{\beta k e^{-d \tau}[\bar{x}(t-\tau)+(\lambda / d)] y(t-\tau)}{\mu[\bar{x}(t-\tau)+(\lambda / d)+y(t-\tau)]} \\
\frac{\mathrm{d} y(t)}{\mathrm{d} t}=\frac{\beta k e^{-d \tau}[\bar{x}(t-\tau)+(\lambda / d)] y(t-\tau)}{\mu[\bar{x}(t-\tau)+(\lambda / d)+y(t-\tau)]}-a y(t),
\end{gathered}
$$

which has two equilibria: the infection-free equilibrium $E_{f}=$ $(0,0,0)^{T}$ and the infected equilibrium $E^{*}=\left(x^{*}, e^{*}, y^{*}\right)^{T}$, where

$$
\begin{gathered}
x^{*}=\frac{\lambda}{d+a e^{d \tau}\left(R_{0}-1\right)}-\frac{\lambda}{d}, \quad e^{*}=\frac{a\left(e^{d \tau}-1\right)}{d} x^{*}, \\
y^{*}=\left(R_{0}-1\right) x^{*} .
\end{gathered}
$$

The following results, Theorems 1 and 2, come from [1].

Theorem 1. If $R_{0}<1$, the infection-free equilibrium $E_{f}$ of the system (5) is globally asymptotically stable.

Theorem 2. If $R_{0}>1$, the chronic infected equilibrium $E^{*}$ of the system (5) is locally asymptotically stable.

The initial conditions for the system (5) are

$$
\begin{gathered}
\left(\varphi_{1}(\theta), \varphi_{2}(\theta), \psi(\theta)\right) \in C_{+}=C\left([-\tau, 0], R_{+0}^{3}\right), \\
\varphi_{k}(0)>0, \quad \psi(0)>0, \quad k=1,2,
\end{gathered}
$$

where $R_{+0}^{3}=\left\{\left(x_{1}, x_{2}, x_{3}\right) \in R^{3}: x_{k}>0, k=1,2,3\right\}$.

It is straightforward to show the following.
Lemma 3. The solution of (5) with an initial condition (6) is nonnegative for all $t \geq 0$.

It is well known that the studies on the dynamical systems not only include the discussion of stabilities, attractivity, and persistence, but also include many dynamical behaviors such as periodic solutions, bifurcations, and chaos. Particularly, the properties of periodic solutions appearing through the Hopf bifurcation in delayed systems are of great interest [57]. In the present paper, our main objective is to investigate the bifurcation phenomena of the modified hepatitis $B$ virus (HBV) model with time delay $\tau$.

This paper is organized as follows. In Section 2, by analyzing the characteristic equation of the linearized system of the system (5) at the equilibria, we discuss the stability of the origin and the positive equilibrium and the existence of the Hopf bifurcations occurring at the chronic infected equilibrium. In Section 3, the formulae determining the direction of the Hopf bifurcations and the stability of bifurcating periodic solutions on the center manifold are obtained by using the normal form theory and the center manifold theorem due to Hassard et al. [8]. To verify the obtained results, some numerical simulations are included in Section 4. The paper ends with a brief discussion.

\section{Stability of Equilibria and Existence of the Hopf Bifurcation}

In this section, we will investigate the stability of the equilibria and the existence of the Hopf bifurcations occurring at the chronic infected equilibrium. Then, it is easy to check that the system (5) has an equilibrium $E_{f}(0,0,0)$ for all nonnegative parameters. The characteristic equation of (5) at $E_{f}$ is

$$
(z+d)^{2}\left(z-\frac{\beta k e^{-d \tau}}{\mu}+a\right)=0
$$

Hence, $E_{f}$ is a saddle with $\operatorname{dim} W^{u}\left(E_{f}\right)=1, \operatorname{dim} W^{s}\left(E_{f}\right)=2$ for $R_{0}<1 ; W^{u}\left(E_{f}\right)$ and $W^{s}\left(E_{f}\right)$ are the local unstable and stable manifolds of $E_{f}$, respectively. $E_{f}$ is locally asymptotically stable for $R_{0}<1$. In fact, $E_{f}$ is globally asymptotically stable for $R_{0}<1$, see $[1,4]$.

Now, we will investigate the stability of the chronic infected equilibrium $E^{*}$. Linearizing the system (5) at $E^{*}$ yields the following linear system:

$$
\begin{aligned}
\frac{\mathrm{d} \bar{x}(t)}{\mathrm{d} t}=-d \bar{x}(t)-\left\{\frac{\beta k y^{*}(t)}{\mu\left[x^{*}(t)+(\lambda / d)+y^{*}(t)\right]}\right. \\
\\
\left.\quad-\frac{\beta k\left[x^{*}(t)+(\lambda / d)\right] y^{*}(t)}{\mu\left[x^{*}(t)+(\lambda / d)+y^{*}(t)\right]^{2}}\right\} \bar{x}(t)
\end{aligned}
$$




$$
\begin{aligned}
& -\left\{\frac{\beta k\left[x^{*}(t)+(\lambda / d)\right]}{\mu\left[x^{*}(t)+(\lambda / d)+y^{*}(t)\right]}\right. \\
& \left.-\frac{\beta k\left[x^{*}(t)+(\lambda / d)\right] y^{*}(t)}{\mu\left[x^{*}(t)+(\lambda / d)+y^{*}(t)\right]^{2}}\right\} y(t), \\
& \frac{\mathrm{d} e(t)}{\mathrm{d} t}=-\mathrm{d} e(t)+\left\{\frac{\beta k y^{*}(t)}{\mu\left[x^{*}(t)+(\lambda / d)+y^{*}(t)\right]}\right. \\
& \left.-\frac{\beta k\left[x^{*}(t)+(\lambda / d)\right] y^{*}(t)}{\mu\left[x^{*}(t)+(\lambda / d)+y^{*}(t)\right]^{2}}\right\} \bar{x}(t) \\
& +\left\{\frac{\beta k\left[x^{*}(t)+(\lambda / d)\right]}{\mu\left[x^{*}(t)+(\lambda / d)+y^{*}(t)\right]}\right. \\
& \left.+\frac{\beta k\left[x^{*}(t)+(\lambda / d)\right] y^{*}(t)}{\mu\left[x^{*}(t)+(\lambda / d)+y^{*}(t)\right]^{2}}\right\} y(t) \\
& -\left\{\frac{\beta k e^{-d \tau} y^{*}(t)}{\mu\left[x^{*}(t)+(\lambda / d)+y^{*}(t)\right]}\right. \\
& \left.-\frac{\beta k e^{-d \tau}\left[x^{*}(t)+(\lambda / d)\right] y^{*}(t)}{\mu\left[x^{*}(t)+(\lambda / d)+y^{*}(t)\right]^{2}}\right\} \bar{x}(t-\tau) \\
& -\left\{\frac{\beta k e^{-d \tau}\left[x^{*}(t)+(\lambda / d)\right]}{\mu\left[x^{*}(t)+(\lambda / d)+y^{*}(t)\right]}\right. \\
& \left.-\frac{\beta k e^{-d \tau}\left[x^{*}(t)+(\lambda / d)\right] y^{*}(t)}{\mu\left[x^{*}(t)+(\lambda / d)+y^{*}(t)\right]^{2}}\right\} y(t-\tau), \\
& \frac{\mathrm{d} y(t)}{\mathrm{d} t}=\left\{\frac{\beta k e^{-d \tau} y^{*}(t)}{\mu\left[x^{*}(t)+(\lambda / d)+y^{*}(t)\right]}\right. \\
& \left.-\frac{\beta k e^{-d \tau}\left[x^{*}(t)+(\lambda / d)\right] y^{*}(t)}{\mu\left[x^{*}(t)+(\lambda / d)+y^{*}(t)\right]^{2}}\right\} \bar{x}(t-\tau) \\
& -\left\{\frac{\beta k e^{-d \tau}\left[x^{*}(t)+(\lambda / d)\right]}{\mu\left[x^{*}(t)+(\lambda / d)+y^{*}(t)\right]}\right. \\
& \left.-\frac{\beta k \mathrm{e}^{-d \tau}\left[x^{*}(t)+(\lambda / d)\right] y^{*}(t)}{\mu\left[x^{*}(t)+(\lambda / d)+y^{*}(t)\right]^{2}}\right\} y(t-\tau) \\
& -a y(t) \text {, }
\end{aligned}
$$

whose characteristic equation reads as

$$
\begin{aligned}
z^{3} & +(2 d+A+a) z^{2}+[d(2 a+A+d)+a A] z+a d(d+A) \\
& =B e^{-z \tau} e^{-d \tau}\left[z^{2}+2 d z+d^{2}\right]
\end{aligned}
$$

with

$$
\begin{aligned}
A= & \frac{\beta k y^{*}(t)}{\mu\left[x^{*}(t)+(\lambda / d)+y^{*}(t)\right]} \\
& -\frac{\beta k\left[x^{*}(t)+(\lambda / d)\right] y^{*}(t)}{\mu\left[x^{*}(t)+(\lambda / d)+y^{*}(t)\right]^{2}}, \\
B= & \frac{\beta k\left[x^{*}(t)+(\lambda / d)\right]}{\mu\left[x^{*}(t)+(\lambda / d)+y^{*}(t)\right]} \\
& -\frac{\beta k\left[x^{*}(t)+(\lambda / d)\right] y^{*}(t)}{\mu\left[x^{*}(t)+(\lambda / d)+y^{*}(t)\right]^{2}} .
\end{aligned}
$$

For $\tau=0$, characteristic equation (10) reduces to

$$
\begin{aligned}
z^{3} & +(2 d+A+a) z^{2}+[d(2 a+A+d)+a A] z+a d(d+A) \\
& =B\left(z^{2}+2 d z+d^{2}\right) .
\end{aligned}
$$

By the Routh-Hurwitz criterion, we know that all the roots of (12) have negative real parts, that is, the chronic infected equilibrium $E^{*}$ is locally asymptotically stable for $\tau=0$. We now give a definition, which can be found in $[2,9]$.

Definition 4. The equilibrium $E^{*}$ is called asymptotically stable if there is an $\varepsilon>0$ such that

$$
\sup _{\tau \leq \theta \leq \infty}\left[\left|\varphi_{1}(\theta)-x_{0}^{*}\right|+\left|\varphi_{2}(\theta)-x_{1}^{*}\right|+\left|\psi(\theta)-y^{*}\right|\right]<\varepsilon
$$

implies that

$$
\lim _{t \rightarrow \infty}\left(x_{0}(t), x_{1}(t), y(t)\right)=\left(x_{0}^{*}, x_{1}^{*}, y^{*}\right),
$$

where $\left(x_{0}(t), x_{1}(t), y(t)\right)$ is the solution of the system (5) with an initial condition (6).

Song et al. [10] investigated the distribution of roots of the following equation:

$$
z^{3}+m_{2} z^{2}+m_{1} z+m_{0}+e^{-z \tau}\left(n_{2} z^{2}+n_{1} z+n_{0}\right)=0,
$$

where $m_{j}, n_{j} \in R(j=0,1,2)$ and $\sum_{j=0}^{2} n_{j}^{2} \neq 0$. When $\tau=0$, (15) reduces to

$$
z^{3}+\left(m_{2}+n_{2}\right) z^{2}+\left(m_{1}+n_{1}\right) z+n_{0}=0 .
$$

Obviously, $i \omega(\omega>0)$ is a root of (16) if and only if $\omega$ satisfies

$$
\begin{aligned}
-\omega^{3} i & -m_{2} \omega^{2}+m_{1} \omega i+m_{0} \\
& +\left(-n_{2} \omega^{2}+n_{1} \omega i+n_{0}\right)(\cos \omega \tau-i \sin \omega \tau)=0 .
\end{aligned}
$$

Separating the real and the imaginary parts, we have $m_{2} \omega^{2}-m_{0}=-n_{2} \omega^{2} \cos \omega \tau+n_{1} \omega \sin \omega \tau+n_{0} \cos \omega \tau$, $-\omega^{3}+m_{1} \omega=-n_{2} \omega^{2} \sin \omega \tau-n_{1} \omega \cos \omega \tau+n_{0} \sin \omega \tau$. 
Squaring the two equations and adding them give

$$
\omega^{6}+p \omega^{4}+q \omega^{2}+r=0,
$$

where $p=m_{2}^{2}-2 m_{1}-n_{2}^{2}, q=m_{1}^{2}-2 m_{0} m_{2}+2 n_{0} n_{2}-n_{1}^{2}$, and $r=m_{0}^{2}-n_{0}^{2}$. Song et al. [10] obtained the following results on the distribution of roots of (15) and (19).

Lemma 5. For the polynomial equation (19),

(i) if $r<0$, then (19) has at least one positive root,

(ii) if $r \geq 0$ and $\Delta=p^{2}-3 q \leq 0$, then (19) has no positive roots,

(iii) if $r \geq 0$ and $\Delta=p^{2}-3 q>0$, then (19) has positive roots if and only if $z_{1}^{*}=(-p+\sqrt{\Delta}) / 3$ and $h\left(z_{1}^{*}\right) \leq 0$, where

$$
h(z)=z^{3}+p z^{2}+q z+r .
$$

Lemma 6. For the transcendental equation (19),

(i) if $r \geq 0$ and $\Delta=p^{2}-3 q \leq 0$, then all roots with positive real parts of (19) have the same sum as those of the polynomial equation (16), for all $\tau$,

(ii) if $r<0$ or $r \geq 0, \Delta=p^{2}-3 q>0, z_{1}^{*}=(-p+\sqrt{\Delta}) / 3>$ 0 and $h\left(z_{1}^{*}\right) \leq 0$, then all roots with positive real parts of (19) have the same sum as those of the polynomial equation (16), for $\tau \in\left[0, \tau_{0}\right.$ ).

From Lemmas 5 and 6, we can have the following lemma.

Lemma 7. (i) The chronic infected equilibrium $E^{*}$ of the system (5) is absolutely stable if and only if the equilibrium $E^{*}$ of the corresponding ordinary differential equation (ODE) system is asymptotically stable, and the characteristic equation (10) has no purely imaginary roots for any $\tau>0$.

(ii) The chronic infected equilibrium $E^{*}$ of the system (5) is conditionally stable if and only if all roots of the characteristic equation (10) have negative real parts at $\tau=0$ such that the characteristic equation (10) has a pair of purely imaginary roots $i \omega_{0}$.

Then, one turns to an investigation of local stability of the chronic infected equilibrium $E^{*}$ in the case of $R_{0}<1$.

Theorem 8. For $R_{0}<1$ holds, there exists a sequence of values for $\tau$ :

$$
\tau_{0}<\tau_{1}<\cdots<\tau_{k}<\cdots,
$$

such that (10) has a pair of purely imaginary roots $i \omega_{0}$ when $\tau=\tau_{k}, k=0,1,2, \ldots$. That is, the chronic infected equilibrium $E^{*}$ of the system (5) is conditionally stable.

Proof. From the above arguments, we know that all roots of characteristic equation (10) have negative real parts at $\tau=$ 0 . Next, we will show that there is a unique pair of purely imaginary roots $i \omega_{0}\left(\omega_{0}>0\right)$ for characteristic equation (10).
Assume that for some $\tau>0, i \omega(\omega>0)$ is a root of (10), which implies that

$$
\begin{array}{r}
-i \omega^{3}-(2 d+A+a) \omega^{2}+[d(2 a+A+d)+a A] i \omega \\
+a d(d+A)=B e^{-i \omega \tau} e^{-d \tau}\left[-\omega^{2}+2 d i \omega+d^{2}\right] .
\end{array}
$$

Note that $A>0, B>0$ because of the positivities of the parameters $\lambda, \mu, \kappa, \beta, d$ and the properties $x^{*}(t)+(\lambda / d) \leq$ $\left[x^{*}(t)+(\lambda / d)+y^{*}(t)\right], y^{*}(t) \leq\left[x^{*}(t)+(\lambda / d)+y^{*}(t)\right]$.

Separating the real and imaginary parts and using Euler's formula give

$$
\begin{aligned}
- & (2 d+A+a) \omega^{2}+a d(d+A) \\
= & B\left(d^{2}-\omega^{2}\right) e^{-d \tau} \cos \omega \tau+2 B d \omega e^{-d \tau} \sin \omega \tau, \\
- & \omega^{3}+[d(2 a+A+d)+a A] \omega \\
= & 2 B d \omega e^{-d \tau} \cos \omega \tau-B\left(d^{2}-\omega^{2}\right) e^{-d \tau} \sin \omega \tau,
\end{aligned}
$$

which is equivalent to

$$
\omega^{6}+p \omega^{4}+q \omega^{2}+r=0,
$$

where

$$
\begin{gathered}
p=(2 d+A+a)^{2}-2[d(2 a+A+d)+a A] \\
=A^{2}+2 d^{2}+a^{2}+2 d A>0, \\
q=[d(2 a+A+d)+a A]^{2}-2(2 d+A+a) a d(d+A), \\
r=-e^{-2 d \tau}\left[B^{2}\left(d^{2}-\omega^{2}\right)^{2}+8 B^{2} d^{2}\right]<0 .
\end{gathered}
$$

In order to solve (24), we first consider the following:

$$
\omega^{6}+p \omega^{4}+q \omega^{2}+r_{0}=0
$$

where

$$
r_{0}=-\left[B^{2}\left(d^{2}-\omega^{2}\right)^{2}+8 B^{2} d^{2}\right]<0
$$

By Lemma 5, there is a unique positive $\omega_{0}$ satisfying (26). From (26), we get the corresponding $\tau_{k}^{\prime}>0$ such that (26) has a pair of purely imaginary roots

$$
\begin{aligned}
& \tau_{k}^{\prime}=\frac{1}{\omega_{0}} \arccos \{\left(a d(d+A)-(2 d+A+a) \omega_{0}^{2}-\omega_{0}^{3}\right. \\
&\left.+[d(2 a+A+d)+a A] \omega_{0}\right) \\
&\left.\times\left(B^{2}\left(d^{2}-\omega^{2}\right)^{2}+8 B^{2} d^{2}\right)^{-1}\right\}+\frac{2 k \pi}{\omega_{0}} \\
&(k=0,1,2, \ldots) .
\end{aligned}
$$

Therefore, by using Rouché's theorem [3], there is a unique positive $\tau_{k}=\tau_{k}^{\prime}+o(1 / k)$ satisfying (26), that is, the characteristic equation (10) has a pair of purely imaginary 
roots of the form $\pm i \omega_{0}$ as $k \rightarrow \infty$. By Lemma 7 , we complete the proof of Theorem 8 .

Next, we turn to show that

$$
\left.\frac{\mathrm{d}(\operatorname{Re} \lambda)}{\mathrm{d} \tau}\right|_{\tau=\tau_{k}}>0 .
$$

This will signify that there exists at least one eigenvalue with positive real part $\tau=\tau_{k}$. We first consider the following:

$$
\begin{aligned}
z^{3} & +(2 d+A+a) z^{2}+[d(2 a+A+d)+a A] z+a d(d+A) \\
& =B e^{-z \tau}\left[z^{2}+2 d z+d^{2}\right] .
\end{aligned}
$$

Differentiating (30) with respect to $\tau$, we have

$$
\begin{aligned}
& \left(\frac{\mathrm{d} \lambda}{\mathrm{d} \tau}\right)^{-1}=\frac{3 z^{2}+2(A+2 d+a) z+[d(2 a+A+d)+a A]}{-B z(d+z)^{2} e^{-z \tau}} \\
& +\frac{\tau e^{-z \tau} B(z+d)^{2}}{-B z(d+z)^{2} e^{-z \tau}}-\frac{2 e^{-z \tau} B(z+d)}{-B z(d+z)^{2} e^{-z \tau}} \\
& =\left(( d + z ) \left\{3 z^{2}+2(A+2 d+a) z\right.\right. \\
& +[d(2 a+A+d)+a A]\}) \\
& \times\left(-B z(d+z)^{3} e^{-z \tau}\right)^{-1} \\
& -\frac{2 e^{-z \tau} B(z+d)^{2}}{-B z(d+z)^{3} e^{-z \tau}}-\frac{\tau}{z} \\
& =-\left(d \left\{3 z^{2}+2(A+2 d+a) z\right.\right. \\
& +[d(2 a+A+d)+a A]\}) \\
& \times\left(( d + z ) \left[z^{3}+(2 d+A+a) z^{2}\right.\right. \\
& +[d(2 a+A+d)+a A] z \\
& +a d(d+A)])^{-1} \\
& -\left(z^{2}-[d(2 a+A+d)+a A] z-2 a d(d+A)\right) \\
& \times\left(( d + z ) \left[z^{3}+(2 d+A+a) z^{2}\right.\right. \\
& +[d(2 a+A+d)+a A] z \\
& +a d(d+A)])^{-1} \\
& -\frac{\tau}{z} .
\end{aligned}
$$

For the sake of simplicity, let $a_{1}=A+2 d+a, a_{2}=d(2 a+$ $A+d)+a A$, and $a_{3}=a d(d+A)$, and (28) can be written as follows:

$$
\begin{aligned}
\left(\frac{\mathrm{d} \lambda}{\mathrm{d} \tau}\right)^{-1}= & -\frac{d\left\{3 z^{2}+2 a_{1} z+a_{2}\right\}}{(d+z)\left[z^{3}+a_{1} z^{2}+a_{2} z+a_{3}\right]} \\
& -\frac{z^{2}-a_{2} z-2 a_{3}}{(d+z)\left[z^{3}+a_{1} z^{2}+a_{2} z+a_{3}\right]}-\frac{\tau}{z} \\
= & \frac{-(3 d+1) z^{2}-\left(2 a_{1} d-a_{2}\right) z-\left(a_{2} d-2 a_{3}\right)}{(d+z)\left[z^{3}+a_{1} z^{2}+a_{2} z+a_{3}\right]}-\frac{\tau}{z} .
\end{aligned}
$$

Therefore,

$$
\begin{aligned}
& \operatorname{sign}\left\{\left.\frac{\mathrm{d}(\operatorname{Re} \lambda)}{\mathrm{d} \tau}\right|_{\tau=\tau_{k}}\right\}=\left.\operatorname{sign}\left\{\operatorname{Re}\left(\frac{\mathrm{d} \lambda}{\mathrm{d} \tau}\right)^{-1}\right\}\right|_{\tau=\tau_{k}} \\
& =\operatorname{sign} \operatorname{Re}\left\{\frac{-(3 d+1) z^{2}-\left(2 a_{1} d-a_{2}\right) z-\left(a_{2} d-2 a_{3}\right)}{(d+z)\left[z^{3}+a_{1} z^{2}+a_{2} z+a_{3}\right]}\right. \\
& \left.-\frac{\tau}{z}\right\} \\
& =\operatorname{sign} \operatorname{Re}\left\{\left(\left(\left[(3 d+1) \omega_{0}^{2}-i\left(2 a_{1} d-a_{2}\right) \omega_{0}\right.\right.\right.\right. \\
& \left.\left.-\left(a_{2} d-2 a_{3}\right)\right]\left(d-i \omega_{0}\right)\right) \\
& \times\left(\left(d^{2}+\omega_{0}^{2}\right)\right. \\
& \left.\left.\times\left[\left(a_{3}^{2}-a_{1} \omega_{0}^{2}\right)^{2}+\left(a_{2} \omega_{0}-\omega_{0}^{3}\right)^{2}\right]\right)^{-1}\right) \\
& \left.\times\left[\left(a_{3}^{2}-a_{1} \omega_{0}^{2}\right)-i\left(a_{2} \omega_{0}-\omega_{0}^{3}\right)\right]\right\} \\
& =\operatorname{sign}\left\{\frac{(3 d+1) \omega_{0}^{6}+p^{2} \omega_{0}^{4}+B d^{2} \omega_{0}^{2}}{\left(d^{2}+\omega_{0}^{2}\right)\left[\left(a_{3}^{2}-a_{1} \omega_{0}^{2}\right)^{2}+\left(a_{2} \omega_{0}-\omega_{0}^{3}\right)^{2}\right]}\right\}>0 .
\end{aligned}
$$

This root of characteristic equation (9) crosses the imaginary axis from the left to the right as $\tau$ continuously varies from a number less than $\tau_{k}$ to one greater than $\tau_{k}$ again by Rouché's theorem [3]. Therefore, the transversality condition holds, and the conditions for Hopf bifurcation [11] are then satisfied at $\tau=\tau_{k}$. In conclusion, we have the following stability and bifurcation results to (5).

Theorem 9. Suppose that $R_{0}<1$ holds. Then, for each fixed $\tau>0$, there exists a sequence of values for $\tau$ :

$$
\tau_{0}<\tau_{1}<\cdots<\tau_{k}<\cdots,
$$

such that the positive equilibrium $E^{*}$ is asymptotically stable when $\tau \in\left[0, \tau_{0}\right)$, and unstable when $\tau>\tau_{0}$. Furthermore, (5) undergoes a Hopf bifurcation at $E^{*}$ when $\tau=\tau_{k}=\tau_{k}^{\prime}+$ $o(1 / k), k=0,1,2, \ldots$, where $\tau_{k}^{\prime}$ is defined by (28). 


\section{Properties of the Hopf Bifurcations}

In this section, we will study the properties of the Hopf bifurcations by using the normal theory and the center manifold theorem due to Hassard et al. [8]. Let $x_{1}=\bar{x}-x^{*}$, $x_{2}=e-e^{*}, x_{3}=y-y^{*}, \tilde{x}_{i}(t)=x_{i}(\tau t), \tau=\tau_{k}+\vartheta$, and $\vartheta \in R=$ $(-\infty, \infty)$. Then, $\vartheta=0$ is the Hopf bifurcation value of system. We drop the tildes for simplification of notations, then the system (5) becomes an functional differential equation in $C\left([-1,0], R^{3}\right)$ as

$$
\dot{x}(t)=L_{\vartheta}\left(x_{t}\right)+f\left(\vartheta, x_{t}\right),
$$

where $C\left([-1,0], R^{3}\right)$ is the Banach space of continuous functions mapping the interval $[-\tau, 0]$ into $R^{3}, x(t)=$ $\left(x_{1}(t), x_{2}(t), x_{3}(t)\right)^{T} \in R^{3}, x_{t}(s)=x(t+s)$ for $s \in[-\tau, 0]$ and $L_{\vartheta}: C \rightarrow R^{3}, f: R \times C \rightarrow R^{3}$ are read, respectively, as

$$
\begin{aligned}
L_{\vartheta}(\phi)=\left(\tau_{k}+\vartheta\right) & \left(\begin{array}{ccc}
-(d+A) & 0 & -B \\
A & -d & B \\
0 & 0 & -a
\end{array}\right)\left(\begin{array}{l}
\phi_{1}(0) \\
\phi_{2}(0) \\
\phi_{3}(0)
\end{array}\right) \\
& +\left(\tau_{k}+\vartheta\right)\left(\begin{array}{ccc}
0 & 0 & 0 \\
-A e^{-d \tau_{k}} & 0 & B e^{-d \tau_{k}} \\
A e^{-d \tau_{k}} & 0 & -B e^{-d \tau_{k}}
\end{array}\right)\left(\begin{array}{l}
\phi_{1}(-1) \\
\phi_{2}(-1) \\
\phi_{3}(-1)
\end{array}\right),
\end{aligned}
$$

$$
\begin{aligned}
f & \left(\vartheta, x_{t}\right) \\
= & \left(\tau_{k}+\vartheta\right) \\
& \times\left(\begin{array}{c}
-\frac{\beta k\left[\phi_{1}(0)+(\lambda / d)\right] \phi_{3}(0)}{\mu\left[\phi_{1}(0)+(\lambda / d)+\phi_{3}(0)\right]} \\
\frac{\beta k\left[\phi_{1}(0)+(\lambda / d)\right] \phi_{3}(0)}{\mu\left[\phi_{1}(0)+(\lambda / d)+\phi_{3}(0)\right]}-\frac{\beta k e^{-d \tau_{k}}\left[\phi_{1}(-1)+(\lambda / d)\right] \phi_{3}(-1)}{\mu\left[\phi_{1}(-1)+(\lambda / d)+\phi_{3}(-1)\right]} \\
\frac{\beta k e^{-d \tau_{k}}\left[\phi_{1}(-1)+(\lambda / d)\right] \phi_{3}(-1)}{\mu\left[\phi_{1}(-1)+(\lambda / d)+\phi_{3}(-1)\right]}
\end{array}\right) .
\end{aligned}
$$

Obviously, $L_{\vartheta}$ is a continuous linear function mapping $C$ into $R^{3}$, by the Riesz representation theorem, there exists a $3 \times 3$ matrix function $\eta(s, \mu)$ of bounded variation for $s \in[-1,0]$, such that

$$
L_{\vartheta}(\phi)=\int_{-1}^{0} \mathrm{~d} \eta(s, \mu) \phi(s) .
$$

In fact, we can choose

$$
\begin{aligned}
\eta(s, \vartheta)= & \left(\tau_{k}+\vartheta\right)\left(\begin{array}{ccc}
-(d+A) & 0 & -B \\
A & -d & B \\
0 & 0 & -a
\end{array}\right) \delta(s) \\
& +\left(\tau_{k}+\vartheta\right)\left(\begin{array}{ccc}
0 & 0 & 0 \\
-A e^{-d \tau_{k}} & 0 & B e^{-d \tau_{k}} \\
A e^{-d \tau_{k}} & 0 & -B e^{-d \tau_{k}}
\end{array}\right) \delta(s+1),
\end{aligned}
$$

where $\delta(\cdot)$ denotes the Dirac delta function.

If $\phi$ is any given function in $C$ and $x(\phi)$ is the unique solution of the linearized equation $\dot{x}(t)=L_{\vartheta}\left(x_{t}\right)$ of (35) with initial function $\phi$ at zero, then the solution operator $T(t)$ : $C \rightarrow C$ is defined by $T(t) \phi=x_{t}(\phi)$, for all $t \geq 0$. It is obvious that $T(t), t \geq 0$, is a strongly continuous semigroup of linear transformation on $[0,+\infty)$ and the infinitesimal generator $A_{\vartheta}$ of $T(t), t \geq 0$, is

$$
A_{\vartheta}= \begin{cases}\frac{\mathrm{d} \phi(s)}{\mathrm{d} s}, & s \in[-1,0) \\ \int_{-1}^{0} \mathrm{~d} \eta(s, \mu) \phi(s), & s=0 .\end{cases}
$$

For $\phi \in C^{1}\left([-1,0], R^{3}\right)$, the space of functions mapping the interval $[-1,0]$ into $R^{3}$ which have a continuous first derivative also defines

$$
R_{\vartheta}(\phi)= \begin{cases}0, & s \in[-1,0) \\ f(\vartheta, \phi), & s=0 .\end{cases}
$$

Then, system (35) is equivalent to

$$
\dot{x}(t)=A_{\vartheta} x_{t}+R_{\vartheta} x_{t} .
$$

For $\psi \in C^{1}\left([-1,0],\left(R^{3}\right)^{*}\right)$, the space of functions mapping interval $[0,1]$ into the three-dimensional row vectors which have continuous first derivative defines

$$
A_{\vartheta}^{*}= \begin{cases}-\frac{\mathrm{d} \psi(s)}{\mathrm{d} s}, & s \in(0,1] \\ \int_{-1}^{0} \mathrm{~d} \eta^{T}(t, 0) \phi(-t), & s=0,\end{cases}
$$

and a bilinear inner product

$$
\langle\psi(\zeta), \phi(s)\rangle=\bar{\psi}(0) \phi(0)-\int_{-1}^{0} \int_{\xi=0}^{s} \bar{\psi}(\xi-s) \mathrm{d} \eta(s) \phi(\xi) \mathrm{d} \xi
$$

where $\eta(s)=\eta(s, 0)$. Then, $A(0)$ and $A^{*}$ are adjoint operators. By the discussion in Section 2, we know that $z$ are eigenvalues of $A(0)$. Hence, they are also eigenvalues of $A^{*}$. We first need to compute the eigenvectors of $A(0)$ and $A^{*}$ corresponding to $i \omega_{0} \tau_{k}$ and $-i \omega_{0} \tau_{k}$, respectively. Suppose that $q(s)=$ $(1, a, b)^{T} e^{i \omega_{0} \tau_{k} s}$ is the eigenvectors of $A(0)$ corresponding to $i \omega_{0} \tau_{k}$, then $A(0) q(s)=i \omega_{0} \tau_{k} q(s)$. Then, from the definition of $A(0)$ and (36), (38), and (39), we have

$$
\begin{aligned}
& \tau_{k}\left(\begin{array}{ccc}
-(d+A) & 0 & -B \\
A & -d & B \\
0 & 0 & -a
\end{array}\right) q(0) \\
& +\tau_{k}\left(\begin{array}{ccc}
0 & 0 & 0 \\
-A e^{-d \tau_{k}} & 0 & B e^{-d \tau_{k}} \\
A e^{-d \tau_{k}} & 0 & -B e^{-d \tau_{k}}
\end{array}\right) q(-1) \\
& \quad=i \omega_{0} \tau_{k} q(0)
\end{aligned}
$$


For $q(-1)=q(0) e^{-i \omega_{0} \tau_{k}}$, then we obtain

$$
\begin{gathered}
\rho=-1+\frac{1}{B}+\frac{A\left(d-i \omega_{0}\right)\left(a+i \omega_{0}\right)}{B\left(\mathrm{~d}^{2}+\omega_{0}^{2}\right)}, \\
\sigma=-\frac{A+d+i \omega_{0}}{B} .
\end{gathered}
$$

Similarly, we can obtain the eigenvector $q^{*}(s)=$ $D\left(1, \rho^{*}, \sigma^{*}\right) e^{i \omega_{0} \tau_{k}}$ of $A^{*}$ corresponding to $-i \omega_{0} \tau_{k}$, where

$$
\begin{gathered}
\rho^{*}=\rho=-1+\frac{1}{B}+\frac{A\left(d-i \omega_{0}\right)\left(a+i \omega_{0}\right)}{B\left(d^{2}+\omega_{0}^{2}\right)}, \\
\sigma^{*}=\sigma=-\frac{A+d+i \omega_{0}}{B} .
\end{gathered}
$$

We need to determine the value of $D$ to ensure that $\left\langle q^{*}(\varsigma), q(s)\right\rangle=1$. By (44), we have

$$
\begin{aligned}
\left\langle q^{*}(\varsigma), q(s)\right\rangle & \\
= & \bar{D}\left(1, \rho^{*}, \sigma^{*}\right)(1, \rho, \sigma)^{T} \\
& -\int_{-1}^{0} \int_{\xi=0}^{s} \bar{D}\left(1, \rho^{*}, \sigma^{*}\right) e^{-i \omega_{0} \tau_{k}(\xi-s)} \mathrm{d} \eta(s)(1, \rho, \sigma)^{T} \\
& \times e^{i \omega_{0} \tau_{k} \xi} \mathrm{d} \xi \\
= & \bar{D}\left[1+|\rho|^{2}+|\sigma|^{2}\right. \\
& \left.\quad-\int_{-1}^{0}\left(1, \rho^{*}, \sigma^{*}\right) s e^{i \omega_{0} \tau_{k} s} \mathrm{~d} \eta(s)(1, \rho, \sigma)^{T}\right] \\
= & \bar{D}\left[1+|\rho|^{2}+|\sigma|^{2}+e^{-d \tau_{k}} e^{-i \omega_{0} \tau_{k}}(A-B \sigma)\left(\bar{\rho}^{*}-\bar{\sigma}^{*}\right)\right] .
\end{aligned}
$$

Therefore, we can choose $D$ as

$$
D=\frac{1}{1+|\rho|^{2}+|\sigma|^{2}+e^{-d \tau_{k}} e^{-i \omega_{0} \tau_{k}}(A-B \sigma)\left(\bar{\rho}^{*}-\bar{\sigma}^{*}\right)} .
$$

Next, we will compute the coordinate to describe the center manifold $C_{0}$ at $\vartheta=0$. Let $x_{t}$ be the solution of (42) when $\vartheta=0$. Define $v(t)=\left\langle q^{*}(\varsigma), x_{t}\right\rangle, \quad W(t, s)=x_{t}(s)-2 \operatorname{Re}\{v(t) q(s)\}$.

On the center manifold $C_{0}$, we have

$$
W(t, s)=W(v(t), \bar{v}(t), s)
$$

where

$$
\begin{aligned}
W & (v(t), \bar{v}(t), s) \\
& =W_{20}(s) \frac{v^{2}}{2}+W_{10}(s) v \bar{v}+W_{02}(s) \frac{\bar{v}^{2}}{2}+\cdots
\end{aligned}
$$

$v$ and $\bar{v}$ are local coordinates for the center manifold $C_{0}$ in the direction of $q^{*}$ and $\bar{q}^{*}$. Note that $W$ is real if $x_{t}$ is real. We only consider real solutions. For solution $x_{t} \in C_{0}$ of (42), since $\vartheta=0$, we have

$$
\begin{aligned}
\dot{v}(t) & =i \omega_{0} \tau_{k} v(t)+\bar{q}^{*}(0) f(0, W(v, \bar{v}, 0)+2 \operatorname{Re}\{v q(s)\}) \\
& :=i \omega_{0} \tau_{k} v(t)+\bar{q}^{*}(0) f_{0}(v, \bar{v})
\end{aligned}
$$

For convenience of calculation, let

$$
\begin{aligned}
\bar{q}^{*} & (0) f_{0}(v, \bar{v}) \\
& =f_{20} \frac{v^{2}}{2}+f_{11}(s) v \bar{v}+f_{02}(s) \frac{\bar{v}^{2}}{2}+f_{21} \frac{v^{2} \bar{v}}{2}+\cdots
\end{aligned}
$$

On the other hand, it follows from (50) and (52) that

$$
\begin{aligned}
x_{t}(s)= & W(v, \bar{v}, 0)+2 \operatorname{Re}\{v q(s)\} \\
= & W_{20}(s) \frac{v^{2}}{2}+W_{10}(s) v \bar{v}+W_{02}(s) \frac{\bar{v}^{2}}{2} \\
& +(1, a, b)^{T} e^{i \omega_{0} \tau_{k} s} v+(1, \bar{a}, \bar{b})^{T} e^{-i \omega_{0} \tau_{k} s} \bar{v}+\cdots .
\end{aligned}
$$

Notice that

$$
\begin{aligned}
\phi_{1}(0)= & q_{1}(0) v+\bar{q}_{1}(0) \bar{v}+W_{20}^{(1)}(0) \frac{v^{2}}{2} \\
& +W_{11}^{(1)}(0) v \bar{v}+W_{02}^{(1)}(0) \frac{\bar{v}^{2}}{2}+\cdots,
\end{aligned}
$$




$$
\begin{aligned}
\phi_{2}(0)= & q_{2}(0) v+\bar{q}_{2}(0) \bar{v}+W_{20}^{(2)}(0) \frac{v^{2}}{2} \\
& +W_{11}^{(2)}(0) v \bar{v}+W_{02}^{(2)}(0) \frac{\bar{v}^{2}}{2}+\cdots, \\
\phi_{3}(0)= & q_{3}(0) v+\bar{q}_{3}(0) \bar{v}+W_{20}^{(3)}(0) \frac{v^{2}}{2} \\
& +W_{11}^{(3)}(0) v \bar{v}+W_{02}^{(3)}(0) \frac{\bar{v}^{2}}{2}+\cdots,
\end{aligned}
$$

$$
\begin{aligned}
\phi_{1}(-1)= & q_{1}(0) v e^{-i \omega_{0}^{*} \tau_{0}^{*}}+\bar{q}_{1}(0) \bar{v} e^{i \omega_{0}^{*} \tau_{0}^{*}} \\
& +W_{20}^{(1)}(-1) \frac{v^{2}}{2}+W_{11}^{(1)}(-1) v \bar{v}+\cdots, \\
\phi_{2}(-1)= & q_{2}(0) v e^{-i \omega_{0}^{*} \tau_{0}^{*}}+\bar{q}_{2}(0) \bar{v} e^{i \omega_{0}^{*} \tau_{0}^{*}} \\
& +W_{20}^{(2)}(-1) \frac{v^{2}}{2}+W_{11}^{(2)}(-1) v \bar{v}+\cdots, \\
\phi_{3}(-1)= & q_{3}(0) v e^{-i \omega_{0}^{*} \tau_{0}^{*}}+\bar{q}_{3}(0) \bar{v} e^{i \omega_{0}^{*} \tau_{0}^{*}} \\
& +W_{20}^{(3)}(-1) \frac{v^{2}}{2}+W_{11}^{(3)}(-1) v \bar{v}+\cdots,
\end{aligned}
$$

Taking (37) into account, we can obtain that

$$
\bar{q}^{*}(0) f_{0}(v, \bar{v})=\bar{q}^{*}(0) f\left(0, x_{t}\right)
$$

$$
\begin{aligned}
& =\tau_{k} \bar{D}\left(1, \bar{\rho}^{*}, \bar{\sigma}^{*}\right)\left(\begin{array}{c}
-\frac{\beta k\left[\phi_{1}(0)+(\lambda / d)\right] \phi_{3}(0)}{\mu\left[\phi_{1}(0)+(\lambda / d)+\phi_{3}(0)\right]} \\
\frac{\beta k\left[\phi_{1}(0)+(\lambda / d)\right] \phi_{3}(0)}{\mu\left[\phi_{1}(0)+(\lambda / d)+\phi_{3}(0)\right]}-\frac{\beta k e^{-d \tau_{k}}\left[\phi_{1}(-1)+(\lambda / d)\right] \phi_{3}(-1)}{\mu\left[\phi_{1}(-1)+(\lambda / d)+\phi_{3}(-1)\right]} \\
\frac{\beta k e^{-d \tau_{k}\left[\phi_{1}(-1)+(\lambda / d)\right] \phi_{3}(-1)}}{\mu\left[\phi_{1}(-1)+(\lambda / d)+\phi_{3}(-1)\right]}
\end{array}\right) \\
& =\tau_{k} \bar{D}\left\{-\frac{\beta k\left[\phi_{1}(0)+(\lambda / d)\right] \phi_{3}(0)}{\mu\left[\phi_{1}(0)+(\lambda / d)+\phi_{3}(0)\right]}+\bar{\rho}^{*} \frac{\beta k\left[\phi_{1}(0)+(\lambda / d)\right] \phi_{3}(0)}{\mu\left[\phi_{1}(0)+(\lambda / d)+\phi_{3}(0)\right]}\right. \\
& \left.-\bar{\rho}^{*} \frac{\beta k e^{-d \tau_{k}}\left[\phi_{1}(-1)+(\lambda / d)\right] \phi_{3}(-1)}{\mu\left[\phi_{1}(-1)+(\lambda / d)+\phi_{3}(-1)\right]}+\bar{\sigma}^{*} \frac{\beta k e^{-d \tau_{k}}\left[\phi_{1}(-1)+(\lambda / d)\right] \phi_{3}(-1)}{\mu\left[\phi_{1}(-1)+(\lambda / d)+\phi_{3}(-1)\right]}\right\} \\
& =\left(\rho^{*}-1\right) \frac{\tau_{k} \bar{D} \beta k}{\mu \Delta_{0}}\left[\frac{\lambda}{d}+q_{1}(0) v+\bar{q}_{1}(0) \bar{v}+W_{20}^{(1)}(0) \frac{v^{2}}{2}+W_{11}^{(1)}(0) v \bar{v}+W_{02}^{(1)}(0) \frac{\bar{v}^{2}}{2}+\cdots\right] \\
& \times\left[q_{3}(0) v+\bar{q}_{3}(0) \bar{v}+W_{20}^{(3)}(0) \frac{v^{2}}{2}+W_{11}^{(3)}(0) v \bar{v}+W_{02}^{(3)}(0) \frac{\bar{v}^{2}}{2}+\cdots\right] \\
& \times\left[\frac{\lambda}{d}+q_{1}(0) v+\bar{q}_{1}(0) \bar{v}+W_{20}^{(1)}(0) \frac{v^{2}}{2}+W_{11}^{(1)}(0) v \bar{v}+W_{02}^{(1)}(0) \frac{\bar{v}^{2}}{2}+\cdots+q_{3}(0) v\right. \\
& \left.+\bar{q}_{3}(0) \bar{v}+W_{20}^{(3)}(0) \frac{v^{2}}{2}+W_{11}^{(3)}(0) v \bar{v}+W_{02}^{(3)}(0) \frac{\bar{v}^{2}}{2}+\cdots\right] \\
& +\left(\sigma^{*}-\rho^{*}\right) \frac{\tau_{k} \bar{D} \beta k e^{-d \tau_{k}}}{\mu \Delta_{-1}} \\
& \times\left[\frac{\lambda}{d}+q_{1}(0) v e^{-i \omega_{0}^{*} \tau_{0}^{*}}+\bar{q}_{1}(0) \bar{v} e^{i \omega_{0}^{*} \tau_{0}^{*}}+W_{20}^{(1)}(0) \frac{v^{2}}{2}+W_{11}^{(1)}(0) v \bar{v}+\cdots\right] \\
& \times\left[q_{3}(0) v e^{-i \omega_{0}^{*} \tau_{0}^{*}}+\bar{q}_{3}(0) \bar{v} e^{i \omega_{0}^{*} \tau_{0}^{*}}+W_{20}^{(3)}(0) \frac{v^{2}}{2}+W_{11}^{(3)}(0) v \bar{v}+W_{02}^{(3)}(0) \frac{\bar{v}^{2}}{2}+\cdots\right]
\end{aligned}
$$


Journal of Applied Mathematics

9

$$
\begin{aligned}
& \times\left[\frac{\lambda}{d}+q_{1}(0) e^{-i \omega_{0}^{*} \tau_{0}^{*}}+\bar{q}_{1}(0) \bar{v} e^{i \omega_{0}^{*} \tau_{0}^{*}}+W_{20}^{(1)}(0) \frac{v^{2}}{2}+W_{11}^{(1)}(0) v \bar{v}+W_{02}^{(1)}(0) \frac{\bar{v}^{2}}{2}+\cdots\right. \\
& \left.\quad+q_{3}(0) v e^{-i \omega_{0}^{*} \tau_{0}^{*}}+\bar{q}_{3}(0) \bar{v} e^{i \omega_{0}^{*} \tau_{0}^{*}}+W_{20}^{(3)}(0) \frac{v^{2}}{2}+W_{11}^{(3)}(0) v \bar{v}+W_{02}^{(3)}(0) \frac{\bar{v}^{2}}{2}+\cdots\right],
\end{aligned}
$$

where

$$
\begin{aligned}
& \Delta_{0}=\mid \frac{\lambda}{d}+q_{1}(0) v+\bar{q}_{1}(0) \bar{v}+W_{20}^{(1)}(0) \frac{v^{2}}{2}+W_{11}^{(1)}(0) v \bar{v} \\
&+W_{02}^{(1)}(0) \frac{\bar{v}^{2}}{2}+\cdots+q_{3}(0) v+\bar{q}_{3}(0) \bar{v} \\
&+W_{20}^{(3)}(0) \frac{v^{2}}{2}+W_{11}^{(3)}(0) v \bar{v}+W_{02}^{(3)}(0) \frac{\bar{v}^{2}}{2}+\left.\cdots\right|^{2} \\
& \Delta_{-1}=\mid \frac{\lambda}{d}+q_{1}(0) v e^{-i \omega_{0}^{*} \tau_{0}^{*}}+\bar{q}_{3}(0) \bar{v}^{i \omega_{0}^{*} \tau_{0}^{*}}+W_{20}^{(1)}(0) \frac{v^{2}}{2} \\
&+W_{11}^{(1)}(0) v \bar{v}+W_{02}^{(1)}(0) \frac{\bar{v}^{2}}{2}+\cdots \\
&+q_{3}(0) v+\bar{q}_{3}(0) \bar{v}+W_{20}^{(3)}(0) \frac{v^{2}}{2} \\
&+W_{11}^{(3)}(0) v \bar{v}+W_{02}^{(3)}(0) \frac{\bar{v}^{2}}{2}+\left.\cdots\right|^{2}
\end{aligned}
$$

Straightforward calculation leads to

$$
\begin{array}{rlr}
\bar{q}^{*}(0) f_{0}(v, \bar{v}) & \times\left[\frac{\lambda}{d}\left(q_{1}(0)+q_{3}(0)\right) q_{3}(0)+\frac{\lambda}{d} q_{1}(0) q_{3}(0)\right. \\
=\left(\rho^{*}-1\right) \frac{\beta k}{\mu \Delta_{0}} & \left.+\frac{1}{2}\left(\frac{\lambda}{d}\right)^{2} W_{20}^{(3)}(-1)\right], \\
\times\left[\frac{\lambda}{d}\left(q_{1}(0)+q_{3}(0)\right) q_{3}(0) v^{2}+\frac{\lambda}{d} q_{1}(0) q_{3}(0) v^{2}\right. & f_{11}=\frac{\lambda}{d}\left(q_{1}(0)+q_{3}(0)\right) q_{3}(0)+2 \operatorname{Re}\left\{\frac{\lambda}{d} q_{1}(0) q_{3}(0)\right\} \\
+\left(\frac{\lambda}{d}\right)^{2} W_{20}^{(3)}(0) \frac{v^{2}}{2}+\frac{\lambda}{d}\left(q_{1}(0)+q_{3}(0)\right) q_{3}(0) v \bar{v} & +\left(\frac{\lambda}{d}\right)^{2} W_{11}^{(3)}(-1)+\frac{\lambda}{d}\left(q_{1}(0)+q_{3}(0)\right) q_{3}(0) \\
+ & +\operatorname{Re}\left\{\frac{\lambda}{d} q_{1}(0) q_{3}(0)\right\} v \bar{v} & +2 \operatorname{Re}\left\{\frac{\lambda}{d} q_{1}(0) q_{3}(0)\right\}+\left(\frac{\lambda}{d}\right)^{2} W_{11}^{(3)}(-1), \\
\left.+\left(\frac{\lambda}{d}\right)^{2} W_{11}^{(3)}(0) v \bar{v}+\cdots\right] & f_{02}=\left(\rho^{*}-1\right) \frac{\beta k}{\mu \Delta}
\end{array}
$$

Comparing the coefficients with (54), we have

$$
\begin{aligned}
f_{20}= & \left(\rho^{*}-1\right) \frac{\beta k}{\mu \Delta_{0}} \\
& \times\left[\frac{\lambda}{d}\left(q_{1}(0)+q_{3}(0)\right) q_{3}(0)+\frac{\lambda}{d} q_{1}(0) q_{3}(0)\right. \\
& \left.+\frac{1}{2}\left(\frac{\lambda}{d}\right)^{2} W_{20}^{(3)}(0)\right] \\
& +\left(\sigma^{*}-\rho^{*}\right) \frac{\tau_{k} \bar{D} \beta k e^{-d \tau_{k}}}{\mu \Delta \Delta_{-1}} \\
& \times\left[\frac{\lambda}{d}\left(q_{1}(0)+q_{3}(0)\right) q_{3}(0)+\frac{\lambda}{d} q_{1}(0) q_{3}(0)\right. \\
& \left.+\frac{1}{2}\left(\frac{\lambda}{d}\right)^{2} W_{20}^{(3)}(-1)\right], \\
f_{11}= & \frac{\lambda}{d}\left(q_{1}(0)+q_{3}(0)\right) q_{3}(0)+2 \operatorname{Re}\left\{\frac{\lambda}{d} q_{1}(0) q_{3}(0)\right\}
\end{aligned}
$$




$$
\begin{aligned}
& \times {\left[\frac{\lambda}{d}\left(\bar{q}_{1}(0)+\bar{q}_{3}(0)\right) \bar{q}_{3}(0)+\frac{\lambda}{d} \bar{q}_{1}(0) \bar{q}_{3}(0)\right.} \\
&\left.+\left(\frac{\lambda}{d}\right)^{2} W_{20}^{(3)}(0) \frac{\bar{v}^{2}}{2}\right] \\
&+\left(\sigma^{*}-\rho^{*}\right) \frac{\tau_{k} \bar{D} \beta k e^{-d \tau_{k}}}{\mu \Delta_{-1}} \\
& \times {\left[\frac{\lambda}{d}\left(\bar{q}_{1}(0)+\bar{q}_{3}(0)\right) \bar{q}_{3}(0)+\frac{\lambda}{d} \bar{q}_{1}(0) \bar{q}_{3}(0)\right.} \\
&\left.+\frac{1}{2}\left(\frac{\lambda}{d}\right)^{2} W_{20}^{(3)}(-1)\right], \\
& f_{21}=\left(\rho^{*}-1\right) \frac{\beta k}{\mu \Delta_{0}} \\
& \times\left[\frac{\lambda}{d}\left(\bar{q}_{1}(0)+\bar{q}_{3}(0)\right) \bar{q}_{3}(0)+\frac{\lambda}{d} \bar{q}_{1}(0) \bar{q}_{3}(0)\right. \\
&\left.+\left(\frac{\lambda}{d}\right)^{2} W_{20}^{(3)}(0) \frac{\bar{v}^{2}}{2}\right] \\
&+\left(\sigma^{*}-\rho^{*}\right) \frac{\tau_{k} \bar{D} \beta k e^{-d \tau_{k}}}{\mu \Delta} \\
& \times+\frac{\lambda}{d}\left(\bar{q}_{1}(0)+\bar{q}_{3}(0)\right) \bar{q}_{3}(0)+\frac{\lambda}{d} \bar{q}_{1}(0) \bar{q}_{3}(0) \\
&\left.+\frac{1}{2}\left(\frac{\lambda}{d}\right)^{2} W_{20}^{(3)}(-1)\right] .
\end{aligned}
$$

Since $W_{20}(s)$ and $W_{11}(s)$ are in $f_{21}$, we still need to compute them. From (40) and (44), we have

$$
\begin{aligned}
\dot{W} & =\dot{x}_{t}-\dot{v} q-\dot{\bar{v}} \bar{q} \\
& = \begin{cases}A W-2 \operatorname{Re}\left\{\bar{q}^{*}(0) f_{0} q(s)\right\}, & s \in[-1,0), \\
A W-2 \operatorname{Re}\left\{\bar{q}^{*}(0) f_{0} q(s)\right\}+f_{0}, & s=0,\end{cases}
\end{aligned}
$$

that is,

$$
\dot{W}=A W+H(v, \bar{v}, s),
$$

where

$$
H(v, \bar{v}, s)=H_{20}(s) \frac{v^{2}}{2}+H_{10}(s) v \bar{v}+H_{02}(s) \frac{\bar{v}^{2}}{2}+\cdots .
$$

Substituting the corresponding series into (67) and comparing the coefficients, we have

$$
\begin{gathered}
\left(A-2 i \omega_{0} \tau_{k}\right) W_{20}(s)=-H_{20}(s), \\
A W_{11}(s)=-H_{11}(s), \ldots .
\end{gathered}
$$

From (67), we know that for $s \in[-1,0)$,

$$
\begin{aligned}
H(v, \bar{v}, s) & =-\bar{q}^{*}(s) f_{0} q(s)-q^{*}(s) \bar{f}_{0} \bar{q}(s) \\
& =-f(v, \bar{v}) q(s)-\bar{f}(v, \bar{v}) \bar{q}(s) .
\end{aligned}
$$

Comparing the coefficients with (68) gives

$$
\begin{aligned}
& H_{20}(s)=-f_{20} q(s)-\bar{f}_{02} \bar{q}(s), \\
& H_{11}(s)=-f_{11} q(s)-\bar{f}_{11} \bar{q}(s) .
\end{aligned}
$$

From the definition of $A$ and (69) and (71), we obtain

$$
\dot{W}_{20}(\mathrm{~s})=2 i \omega_{0} \tau_{k} W_{20}(s)+f_{20} q(s)+\bar{f}_{02} \bar{q}(s) .
$$

For $q(s)=(1, \rho, \sigma)^{T} e^{i \omega_{0} \tau_{k} s}$, hence

$$
\begin{aligned}
W_{20}(s)= & \frac{i f_{20}}{\omega_{0} \tau_{k}} q(0) e^{i \omega_{0} \tau_{k} s} \\
& +\frac{i \bar{f}_{02}}{3 \omega_{0} \tau_{k}} q(0) e^{-i \omega_{0} \tau_{k} s} \bar{q}(s)+E_{1} e^{2 i \omega_{0} \tau_{k} s},
\end{aligned}
$$

where $E_{1}=\left(E_{1}^{(1)}, E_{1}^{(2)}, E_{1}^{(3)}\right)$ is a constant vector. Similarly, we know that

$$
W_{11}(s)=-\frac{i f_{11}}{\omega_{0} \tau_{k}} q(0) e^{i \omega_{0} \tau_{k} s}+\frac{i \bar{f}_{11}}{\omega_{0} \tau_{k}} q(0) e^{-i \omega_{0} \tau_{k} s} \bar{q}(s)+E_{2},
$$

where $E_{2}=\left(E_{2}^{(1)}, E_{2}^{(2)}, E_{2}^{(3)}\right)$ is a constant vector.

In what follows, we will calculate $E_{1}$ and $E_{2}$. From the definition of $A$ and (69), we have

$$
\begin{gathered}
\int_{-1}^{0} \mathrm{~d} \eta^{T}(s) W_{20}(s)=2 i \omega_{0} \tau_{k} W_{20}(0)-H_{20}(0), \\
\int_{-1}^{0} \mathrm{~d} \eta^{T}(s) W_{11}(s)=-H_{11}(0),
\end{gathered}
$$

where $\eta(s)=\eta(s, 0)$. When $s=0$, we obtain that

$$
\begin{aligned}
H(v, \bar{v}, 0) & =-2 \operatorname{Re}\left\{\bar{q}^{*}(0) f_{0} q(0)\right\}+f_{0} \\
& =-\bar{q}^{*}(0) f_{0} q(0)-q^{*}(0) \bar{f}_{0} \bar{q}(0)+f_{0},
\end{aligned}
$$

that is,

$$
\begin{aligned}
H_{20}(s) & \frac{v^{2}}{2}+H_{11}(s) v \bar{v}+H_{02}(s) \frac{\bar{v}^{2}}{2}+\cdots \\
= & -q(0)\left(f_{20} \frac{v^{2}}{2}+f_{11}(s) v \bar{v}+f_{02}(s) \frac{\bar{v}^{2}}{2}+f_{21} \frac{v^{2} \bar{v}}{2} \cdots\right) \\
& -\bar{q}(0)\left(\bar{f}_{20} \frac{v^{2}}{2}+\bar{f}_{11}(s) v \bar{v}+\bar{f}_{02}(s) \frac{\bar{v}^{2}}{2}+f_{21} \frac{v^{2} \bar{v}}{2} \cdots\right) \\
& +f_{0},
\end{aligned}
$$

with

$$
f_{0}=\tau_{k}\left(\begin{array}{c}
-\frac{\beta k\left[\phi_{1}(0)+(\lambda / d)\right] \phi_{3}(0)}{\mu\left[\phi_{1}(0)+(\lambda / d)+\phi_{3}(0)\right]} \\
\frac{\beta k\left[\phi_{1}(0)+(\lambda / d)\right] \phi_{3}(0)}{\mu\left[\phi_{1}(0)+(\lambda / d)+\phi_{3}(0)\right]}-\frac{\beta k e^{-d \tau_{k}}\left[\phi_{1}(-1)+(\lambda / d)\right] \phi_{3}(-1)}{\mu\left[\phi_{1}(-1)+(\lambda / d)+\phi_{3}(-1)\right]} \\
\frac{\beta k e^{-d \tau_{k}}\left[\phi_{1}(-1)+(\lambda / d)\right] \phi_{3}(-1)}{\mu\left[\phi_{1}(-1)+(\lambda / d)+\phi_{3}(-1)\right]}
\end{array}\right) .
$$


From (50), we have

$$
f_{0}=\left(\begin{array}{c}
f_{v^{2}}^{1} \\
f_{v^{2}}^{2} \\
f_{v^{2}}^{3}
\end{array}\right) v^{2}+\left(\begin{array}{c}
f_{v \bar{v}}^{1} \\
f_{v \bar{v}}^{2} \\
f_{v \bar{v}}^{3}
\end{array}\right) v \bar{v}+\cdots
$$

where

$$
\begin{aligned}
f_{v^{2}}^{1}=-\frac{\beta k}{\mu \Delta_{0}}[ & \frac{\lambda}{d}\left(q_{1}(0)+q_{3}(0)\right) q_{3}(0)+\frac{\lambda}{d} q_{1}(0) q_{3}(0) \\
& +\frac{1}{2}\left(\frac{\lambda}{d}\right)^{2} \\
& \times\left(\frac{i f_{20}}{\omega_{0} \tau_{k}} q(0) e^{i \omega_{0} \tau_{k} s}+\frac{i \bar{f}_{02}}{3 \omega_{0} \tau_{k}} q(0) e^{-i \omega_{0} \tau_{k} s} \bar{q}(s)\right. \\
& \left.\left.+E_{1} e^{2 i \omega_{0} \tau_{k} s}\right)\right]
\end{aligned}
$$

$$
\begin{aligned}
f_{v^{2}}^{2}=\frac{\beta k}{\mu \Delta_{0}}\left[\frac{\lambda}{d}\left(q_{1}(0)+q_{3}(0)\right) q_{3}(0)+\frac{\lambda}{d} q_{1}(0) q_{3}(0)\right. \\
+\frac{1}{2}\left(\frac{\lambda}{d}\right)^{2} \\
\quad \times\left(\frac{i f_{20}}{\omega_{0} \tau_{k}} q(0) e^{i \omega_{0} \tau_{k} s}+\frac{i \bar{f}_{02}}{3 \omega_{0} \tau_{k}} q(0) e^{-i \omega_{0} \tau_{k} s} \bar{q}(s)\right. \\
+\frac{\lambda}{d}\left(q_{1}(0)+E_{1}(0)\right) q_{3}(0) \\
+2 \operatorname{Re}\left\{\frac{\lambda}{d} q_{1}(0) q_{3}(0)\right\}+\left(\frac{\lambda}{d}\right)^{2} W_{11}^{(3)}(-1)
\end{aligned}
$$

$$
\begin{gathered}
+\frac{\lambda}{d}\left(q_{1}(0)+q_{3}(0)\right) q_{3}(0) \\
+2 \operatorname{Re}\left\{\frac{\lambda}{d} q_{1}(0) q_{3}(0)\right\}+\left(\frac{\lambda}{d}\right)^{2} W_{11}^{(3)}(-1) \\
f_{v^{2}=-}^{3} \frac{\beta k e^{-d \tau_{k}}}{\mu \Delta_{-1}}\left[\frac{\lambda}{d}\left(q_{1}(0)+q_{3}(0)\right) q_{3}(0)+\frac{\lambda}{d} q_{1}(0) q_{3}(0)\right. \\
+\frac{1}{2}\left(\frac{\lambda}{d}\right)^{2} \\
\times\left(\frac{i f_{20}}{\omega_{0} \tau_{k}} q(0) e^{i \omega_{0} \tau_{k} s}\right. \\
+\frac{i \bar{f}_{02}}{3 \omega_{0} \tau_{k}} q(0) e^{-i \omega_{0} \tau_{k} s} \bar{q}(s) \\
\left.\left.+E_{1} e^{2 i \omega_{0} \tau_{k} s}\right)\right]
\end{gathered}
$$

By (70) and (71), we have

$$
\begin{aligned}
& H_{20}(s)=-f_{20} q(s)-\bar{f}_{02} \bar{q}(s)+f_{v^{2}}, \\
& H_{11}(s)=-f_{11} q(s)-\bar{f}_{11} \bar{q}(s)+f_{v \bar{v}} .
\end{aligned}
$$

For $i \omega_{0} \tau_{k}$ is the eigenvalue of $A(0)$ and $q(0)$ is the corresponding eigenvector, we obtain

$$
\begin{gathered}
{\left[i \omega_{0} \tau_{k} I-\int_{-1}^{0} e^{i \omega_{0} \tau_{k} s} \mathrm{~d} \eta(s)\right] q(0)=0,} \\
{\left[-i \omega_{0} \tau_{k} I-\int_{-1}^{0} e^{-i \omega_{0} \tau_{k} s} \mathrm{~d} \eta(s)\right] \bar{q}(0)=0 .}
\end{gathered}
$$

So substituting (66) and (73) into (68), we obtain

$$
\left[2 i \omega_{0} \tau_{k} I-\int_{-1}^{0} e^{2 i \omega_{0} \tau_{k} s} \mathrm{~d} \eta(\mathrm{s})\right] E_{1}=\left(\begin{array}{c}
f_{v^{2}}^{1} \\
f_{v^{2}}^{2} \\
f_{v^{2}}^{3}
\end{array}\right)
$$

that is,

$$
\left(\begin{array}{ccc}
2 i \omega_{0} \tau_{k}+(d+A) & 0 & B \\
A\left(e^{-d \tau_{k}} e^{-2 i \omega_{0} \tau_{k}}-1\right) & 2 i \omega_{0} \tau_{k}+d & B\left(e^{-d \tau_{k}} e^{-2 i \omega_{0} \tau_{k}}-1\right) \\
-A e^{-d \tau_{k}} e^{-2 i \omega_{0} \tau_{k}} & 0 & 2 i \omega_{0} \tau_{k}+a+B e^{-d \tau_{k}} e^{-2 i \omega_{0} \tau_{k}}
\end{array}\right) E_{1}=\left(\begin{array}{c}
f_{v^{2}}^{1} \\
f_{v^{2}}^{2} \\
f_{v^{2}}^{3}
\end{array}\right)
$$




$$
\begin{gathered}
E_{1}^{(1)}=\frac{1}{M_{1}} \operatorname{det}\left(\begin{array}{ccc}
f_{v^{2}}^{1} & 0 & B \\
f_{v^{2}}^{2} & 2 i \omega_{0} \tau_{k}+d & B\left(e^{-d \tau_{k}} e^{-2 i \omega_{0} \tau_{k}}-1\right) \\
f_{v^{2}}^{3} & 0 & 2 i \omega_{0} \tau_{k}+a+B e^{-d \tau_{k}} e^{-2 i \omega_{0} \tau_{k}}
\end{array}\right), \\
E_{1}^{(2)}=\frac{1}{M_{1}} \operatorname{det}\left(\begin{array}{ccc}
2 i \omega_{0} \tau_{k}+(d+A) & f_{v^{2}}^{1} & B \\
A\left(e^{-d \tau_{k}} e^{-2 i \omega_{0} \tau_{k}}-1\right) & f_{v^{2}}^{2} & B\left(e^{-d \tau_{k}} e^{-2 i \omega_{0} \tau_{k}}-1\right) \\
-A e^{-d \tau_{k}} e^{-2 i \omega_{0} \tau_{k}} & f_{v^{2}}^{3} & 2 i \omega_{0} \tau_{k}+a+B e^{-d \tau_{k}} e^{-2 i \omega_{0} \tau_{k}}
\end{array}\right), \\
E_{1}^{(3)}=\frac{1}{M_{1}} \operatorname{det}\left(\begin{array}{ccc}
2 i \omega_{0} \tau_{k}+(d+A) & 0 & f_{v^{2}}^{1} \\
A\left(e^{-d \tau_{k}} e^{-2 i \omega_{0} \tau_{k}}-1\right) & 2 i \omega_{0} \tau_{k}+d & f_{v^{2}}^{2} \\
-A e^{-d \tau_{k}} e^{-2 i \omega_{0} \tau_{k}} & 0 & f_{v^{2}}^{3}
\end{array}\right),
\end{gathered}
$$

where

$$
M_{1}=\operatorname{det}\left(\begin{array}{ccc}
2 i \omega_{0} \tau_{k}+(d+A) & 0 & B \\
A\left(e^{-d \tau_{k}} e^{-2 i \omega_{0} \tau_{k}}-1\right) & 2 i \omega_{0} \tau_{k}+d & B\left(e^{-d \tau_{k}} e^{-2 i \omega_{0} \tau_{k}}-1\right) \\
-A e^{-d \tau_{k}} e^{-2 i \omega_{0} \tau_{k}} & 0 & 2 i \omega_{0} \tau_{k}+a+B e^{-d \tau_{k}} e^{-2 i \omega_{0} \tau_{k}}
\end{array}\right)
$$

Similarly, substituting (67) and (74) into (69), we get

$$
\begin{gathered}
E_{2}^{(1)}=\frac{1}{M_{2}} \operatorname{det}\left(\begin{array}{ccc}
f_{v \bar{v}}^{1} & 0 & B \\
f_{v \bar{v}}^{2} & d & B\left(e^{-d \tau_{k}} e^{-i \omega_{0} \tau_{k}}-1\right) \\
f_{\bar{v}}^{3} & 0 & a+B e^{-d \tau_{k}} e^{-i \omega_{0} \tau_{k}}
\end{array}\right), \\
E_{2}^{(2)}=\frac{1}{M_{2}} \operatorname{det}\left(\begin{array}{ccc}
(d+A) & f_{v \bar{v}}^{1} & B \\
A\left(e^{-d \tau_{k}} e^{-i \omega_{0} \tau_{k}}-1\right) & f_{v \bar{v}}^{2} & B\left(e^{-d \tau_{k}} e^{-i \omega_{0} \tau_{k}}-1\right) \\
-A e^{-d \tau_{k}} e^{-i \omega_{0} \tau_{k}} & f_{v \bar{v}}^{3} & a+B e^{-d \tau_{k}} e^{-i \omega_{0} \tau_{k}}
\end{array}\right), \\
E_{2}^{(3)}=\frac{1}{M_{2}} \operatorname{det}\left(\begin{array}{cccc}
(d+A) & 0 & f_{v \bar{v}}^{1} \\
A\left(e^{-d \tau_{k}} e^{-i \omega_{0} \tau_{k}}-1\right) & d & f_{v \bar{v}}^{2} \\
-A e^{-d \tau_{k}} e^{-i \omega_{0} \tau_{k}} & 0 & f_{v \bar{v}}^{3}
\end{array}\right),
\end{gathered}
$$




$$
M_{2}=\operatorname{det}\left(\begin{array}{ccc}
(d+A) & 0 & B \\
A\left(e^{-d \tau_{k}} e^{-i \omega_{0} \tau_{k}}-1\right) & d & B\left(e^{-d \tau_{k}} e^{-i \omega_{0} \tau_{k}}-1\right) \\
-A e^{-d \tau_{k}} e^{-i \omega_{0} \tau_{k}} & 0 & a+B e^{-d \tau_{k}} e^{-i \omega_{0} \tau_{k}}
\end{array}\right) .
$$

Thus, we can determine $W_{20}(s)$ and $W_{11}(s)$ from (66) and (67). Furthermore, we can compute $f_{21}$ by (55). Thus, we can compute the following values:

$$
\begin{gathered}
c_{(1)}(0)=\frac{i}{2 \omega_{0} \tau_{k}}\left(f_{20} f_{11}-2\left|f_{11}\right|^{2}-\frac{\left|f_{02}\right|^{2}}{3}\right)+\frac{f_{21}}{2}, \\
\vartheta_{2}=-\frac{\operatorname{Im}\left\{c_{1}(0)\right\}}{\operatorname{Re}\left\{\lambda^{\prime}\left(\tau_{k}\right)\right\}}, \\
\sigma_{2}=2 \operatorname{Re}\left\{c_{(1)}(0)\right\}, \\
T_{2}=-\frac{\operatorname{Im}\left\{c_{1}(0)\right\}+\vartheta_{2} \operatorname{Re}\left\{\lambda^{\prime}\left(\tau_{k}\right)\right\}}{\omega_{0} \tau_{k}} .
\end{gathered}
$$

Then from [8], we can give the properties of bifurcating periodic solution in the center manifold at the critical values $\tau_{k}$. More specifically, we have the following theorem.

Theorem 10. The properties of the Hopf bifurcation are determined by the values in (75).

(1) $\vartheta_{2}$ determines the directions of the Hopf bifurcation: if $\vartheta_{2}>0\left(\vartheta_{2}<0\right)$, then the Hopf bifurcation is supercritical (subcritical), and the bifurcating periodic solutions exist for $\tau>\tau_{k}\left(\tau<\tau_{k}\right)$.

(2) $\vartheta_{2}$ determines the stability of the bifurcating periodic solutions: the bifurcating periodic solutions are stable (unstable) if $\vartheta_{2}>0\left(\vartheta_{2}<0\right)$.

(3) $T_{2}$ determines the period of the bifurcating periodic solutions: the period increases (decreases) if $T_{2}>$ $0\left(T_{2}<0\right)$.

\section{Numerical Simulations}

As an example, we consider the system given in [1] with $\lambda=$ $1.5 \times 10^{5}, d=0.011, \beta=9.8 \times 10^{-7}, k=150, a=0.1$, and $\mu=0.67$, then, there is a chronic infected equilibrium $E^{*}$ which satisfies the conditions indicated in Theorem 9. When $\tau=0$, the chronic infected equilibrium $E^{*}=(-1.3515 \times$ $\left.10^{7},-1.0120 \times 10^{5}, 1.2090 \times 10^{5}\right)$ is asymptotically stable (see Figure 1(a) which is the amplified part of Figure 1(b) during time interval $[0,30])$. It follows from the discussion in Section 2 that $\omega_{0}=0.6029, \tau_{0}=8.1786$ and $\lambda\left(\tau_{0}\right)=0.2103-$ $0.3942 i$. From the formulae (92) in Section 3, it follows that $c_{1}(0)=-0.1052-0.3061 i, \vartheta_{2}=8.2963>0, \sigma_{2}=-0.2103<0$, and $T_{2}=1.6737>0$. Thus, the chronic infected equilibrium $E^{*}$ is asymptotically stable when $0 \leq \tau<\tau_{0}$ as illustrated by the computer simulations (see Figures 2 and 3 ).

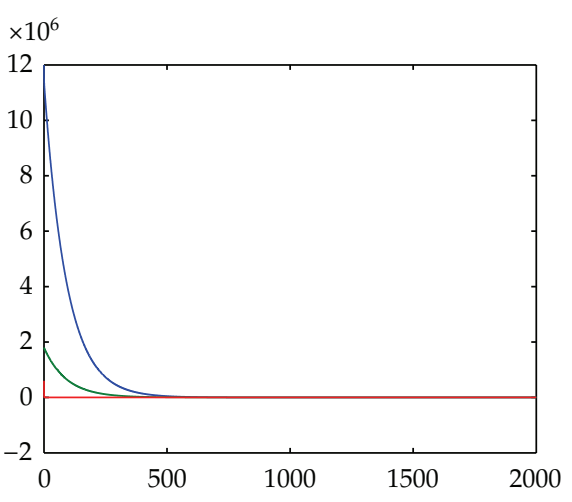

(a)

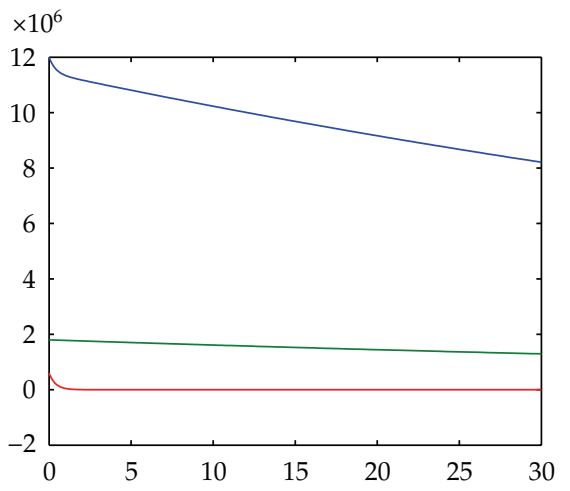

(b)

FIgURE 1: The response of the system (5) initial data $(1.2 e 7,1.8 e 6,6 e 5)^{T}$, when $\tau=0$.

When $\tau$ passes through the critical value $\tau_{0}, E^{*}$ loses its stability, and a Hopf bifurcation occurs, that is, a family of periodic solutions bifurcate from $E^{*}$. Since $\vartheta_{2}$ and $\sigma_{2}=$ $-0.2103<0$, the Hopf bifurcation is supercritical, and the direction of the bifurcation is $\tau>\tau_{0}$, and these bifurcating periodic solutions from $E^{*}$ at $\tau_{0}$ are stable, which are depicted in Figures 2 and 3.

In Figure 4, we try to reflect the changes of stability of chronic infected equilibrium $E^{*}$ as $\tau$ increases from 0 to 9 . In Figure 4 , each vertical blue strip corresponds to component of $\bar{x}(t), e(t)$, and $y(t)$ at each $\tau$, respectively. From Figure 3, we see that if $\tau \in(0,8.17864293)$, approximately, the vertical amplitudes of $\bar{x}(t), e(t)$, and $y(t)$ are as small as a point, suggesting that $E^{*}$ is asymptotically stable; if $\tau$ increases, the vertical amplitudes of $\bar{x}(t), e(t)$, and $y(t)$ will become larger and larger, showing that $E^{*}$ becomes more and more unstable. In particular, if $\tau=8.1787$, points are well distributed 


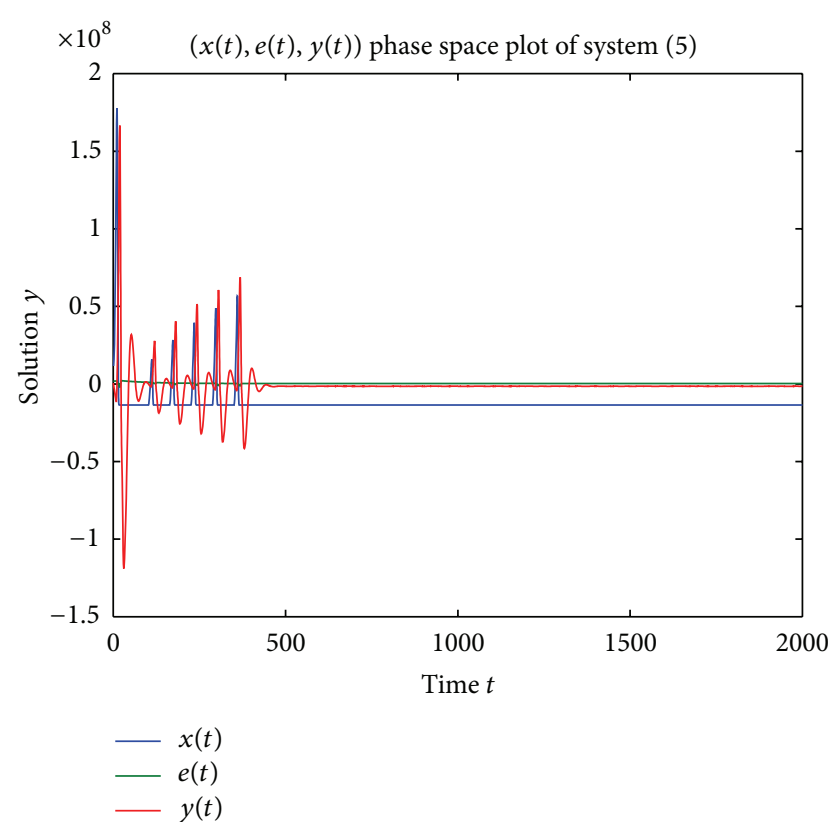

Figure 2: The response of the system (5) initial data $(1.2 e 7,1.8 e 6,6 e 5)^{T}$, when $\tau=8.1786<\tau_{0}$.

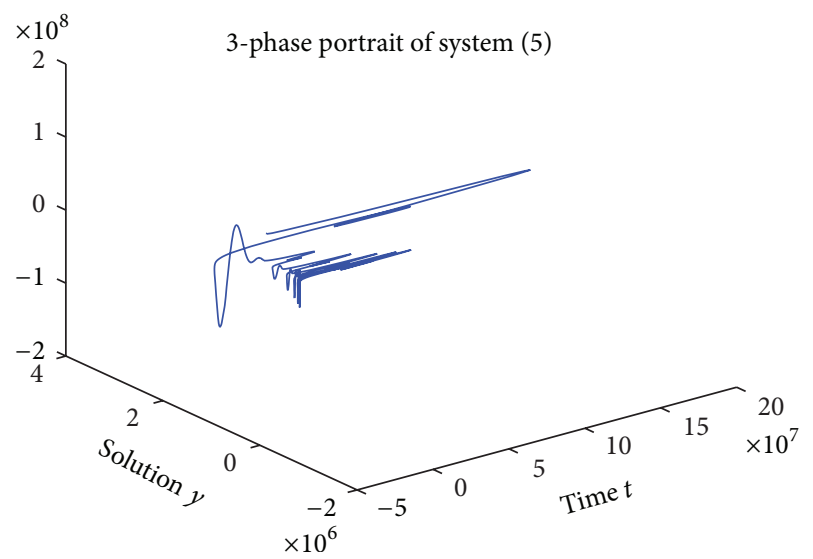

FIGURE 3: 3-phase portrait of the system (5) initial data $(1.2 e 7,1.8 e 6,6 e 5)^{T}$, when $\tau=8.1786<\tau_{0}$.

around the positive equilibrium, and their amplitudes are all equivalent. This shows that periodic solution near positive equilibrium may occur.

\section{Conclusions}

In this paper, a delay differential equation of hepatitis $B$ virus infection is formulated. We analyzed the stability of the equilibria; a sufficient condition was given to guarantee the global stability of the origin. Local stability of the chronic infected equilibrium was considered. By choosing time delay $\tau$ as a bifurcated parameter, a sufficient condition has been presented for checking the existence of the Hopf bifurcation. The explicit formulae determining the direction, stability, and other properties of bifurcating periodic solutions were given

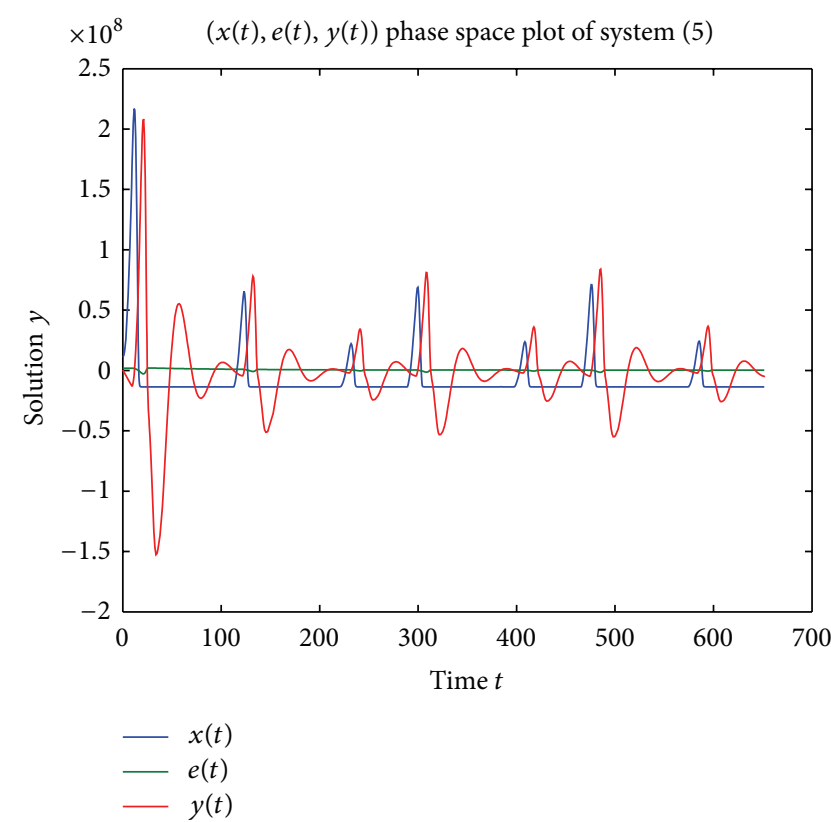

Figure 4: The response of the system (5) initial data $(1.2 e 7,1.8 e 6,6 e 5)^{T}$, when $\tau=8.21>\tau_{0}$.

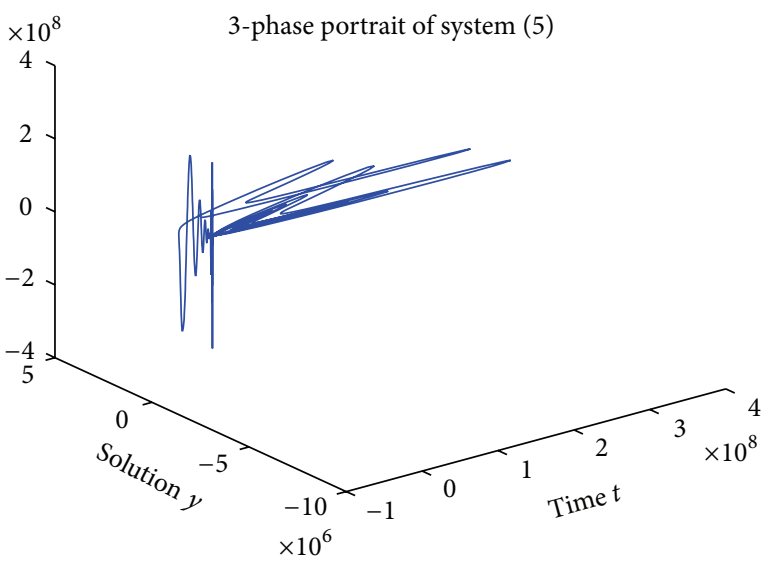

FIgURE 5: 3-phase portrait of the system (5) initial data $(1.2 e 7,1.8 e 6,6 e 5)^{T}$, when $\tau=8.21>\tau_{0}$.

by using the normal form theory and the center manifold theorem. Some numerical simulations were performed to support the analytical results found. Although bifurcations in a population dynamics with delay has been investigated by many researchers. However, to the best of our knowledge, there are few papers on the bifurcation of delay differential equation of hepatitis B virus infection dynamics with delay.

\section{Acknowledgments}

This work was partially supported by the National Natural Science Foundation of China (Grant nos. 11201179 and 11026112). This work was partially supported by the Foundation of University of Jinan (Grant no. XKY1133) and the Shandong Province Young and Middle-Aged Scientists 
Research Awards Fund (Grant no. BS2011SF001). The authors would like to thank the reviewers for their careful reading of the paper and for their valuable comments and suggestions.

\section{References}

[1] S. A. Gourley, Y. Kuang, and J. D. Nagy, "Dynamics of a delay differential equation model of hepatitis B virus infection," Journal of Biological Dynamics, vol. 2, no. 2, pp. 140-153, 2008.

[2] Y. Zheng, L. Min, Y. Ji, Y. Su, and Y. Kuang, "Global stability of endemic equilibrium point of basic virus infection model with application to HBV infection," Journal of Systems Science \& Complexity, vol. 23, no. 6, pp. 1221-1230, 2010.

[3] S. Hews, S. Eikenberry, J. D. Nagy, and Y. Kuang, "Rich dynamics of a hepatitis B viral infection model with logistic hepatocyte growth," Journal of Mathematical Biology, vol. 60, no. 4, pp. 573-590, 2010.

[4] B.-Z. Guo and L.-M. Cai, "A note for the global stability of a delay differential equation of hepatitis B virus infection," Mathematical Biosciences and Engineering, vol. 8, no. 3, pp. 689694, 2011.

[5] J. K. Hale, Theory of Functional Differential Equations, Springer, New York, NY, USA, 2nd edition, 1977.

[6] J. Wei and S. Ruan, "Stability and bifurcation in a neural network model with two delays," Physica D, vol. 130, no. 3-4, pp. 255-272, 1999.

[7] X.-P. Yan, "Hopf bifurcation and stability for a delayed trineuron network model," Journal of Computational and Applied Mathematics, vol. 196, no. 2, pp. 579-595, 2006.

[8] B. D. Hassard, N. D. Kazarinoff, and Y. H. Wan, Theory and Applications of Hopf Bifurcation, vol. 41, Cambridge University Press, Cambridge, UK, 1981.

[9] Y. Kuang, Delay Differential Equations with Applications in Population Dynamics, vol. 191, Academic Press, Boston, Mass, USA, 1993.

[10] Y. Song, M. Han, and J. Wei, "Stability and Hopf bifurcation analysis on a simplified BAM neural network with delays," Physica D, vol. 200, no. 3-4, pp. 185-204, 2005.

[11] J. K. Hale and S. Lunel, Introduction to Functional-Differential Equations, vol. 99, Springer, New York, NY, USA, 1993. 


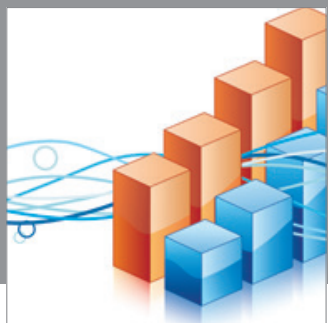

Advances in

Operations Research

mansans

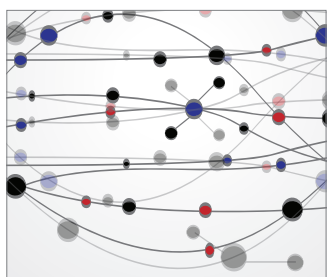

The Scientific World Journal
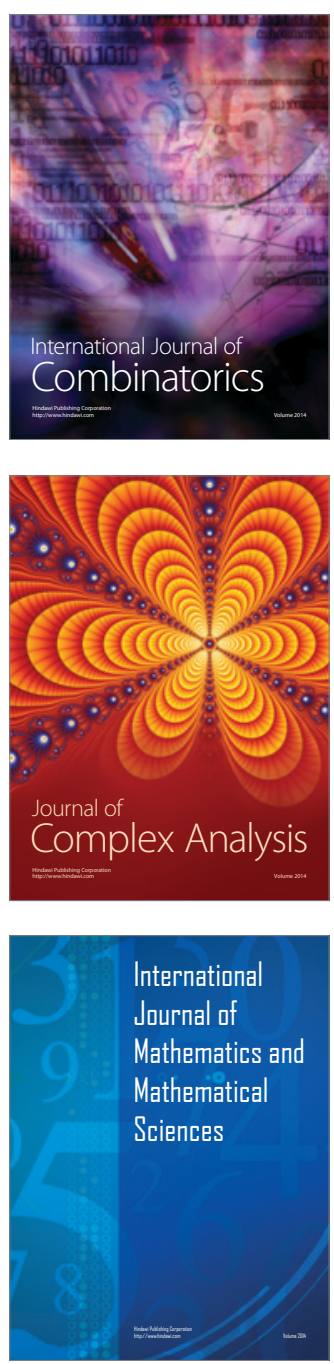
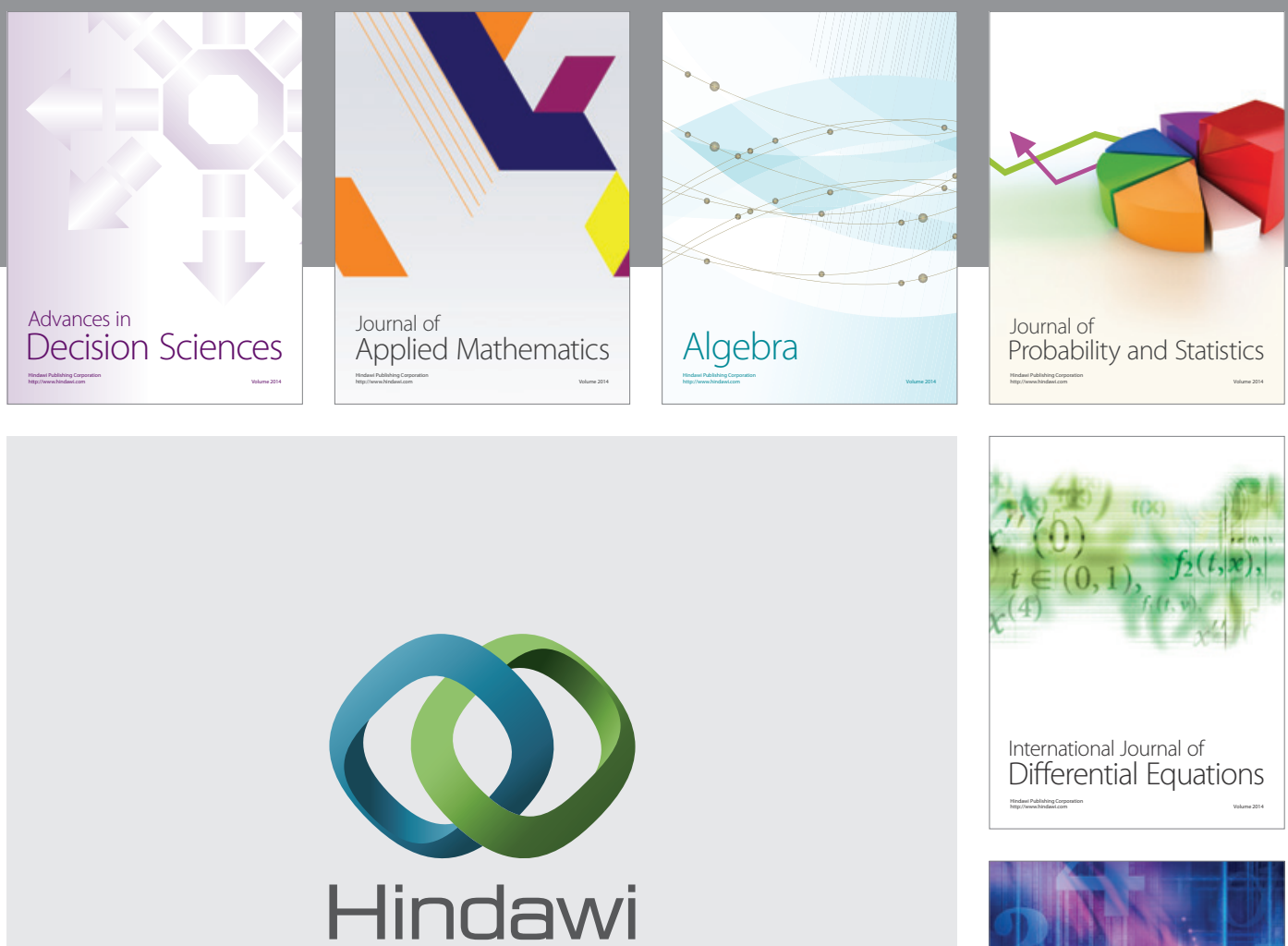

Submit your manuscripts at http://www.hindawi.com
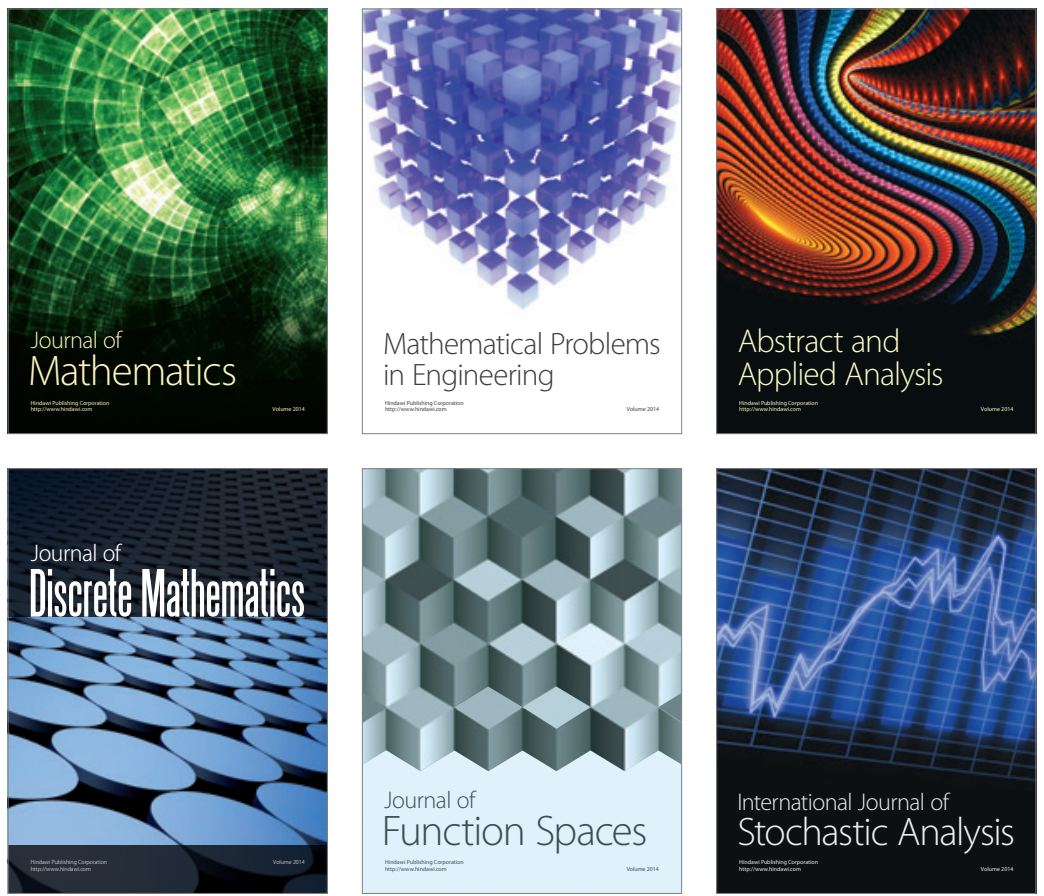

Journal of

Function Spaces

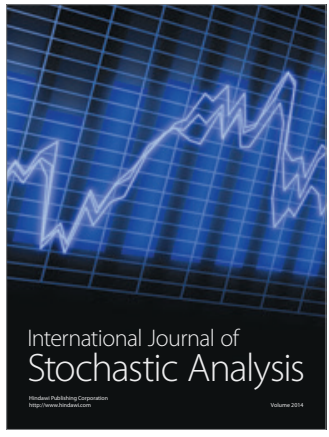

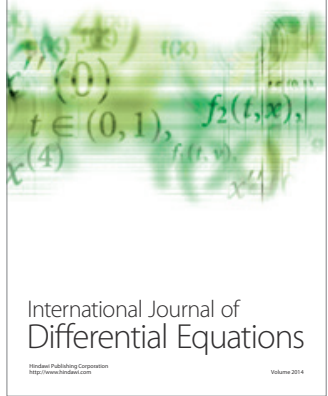
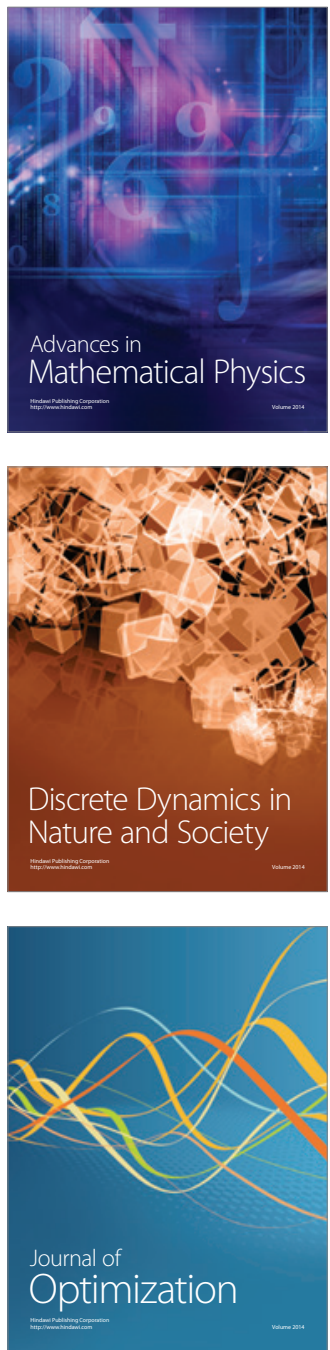